\title{
Toric Spines at a Site of Learning
}

\author{
Daniel Sanculi, ${ }^{1{ }^{*}}$ Katherine E. Pannoni, ${ }^{1 *}$ EEric A. Bushong, ${ }^{2}$ Michael Crump, ${ }^{1}$ Michelle Sung, ${ }^{1}$ (D) Vyoma \\ Popat, ${ }^{1}$ Camilia Zaher, ${ }^{1}$ Emma Hicks, ${ }^{1}$ Ashley Song, ${ }^{1}$ Nikan Mofakham, ${ }^{1}$ ○Peining Li, ${ }^{1}$ Evan G. \\ Antzoulatos, ${ }^{1}$ Diasynou Fioravante, ${ }^{1}$ Mark H. Ellisman, ${ }^{2}$ and ${ }^{-W}$ William M. DeBello ${ }^{1}$
}

https://doi.org/10.1523/ENEURO.0197-19.2019

${ }^{1}$ Center for Neuroscience, University of California, Davis, CA 95618 and ${ }^{2}$ National Center for Molecular Imaging Research, University of California, La Jolla, CA 92093

\begin{abstract}
We discovered a new type of dendritic spine. It is found on space-specific neurons in the barn owl inferior colliculus, a site of experience-dependent plasticity. Connectomic analysis revealed dendritic protrusions of unusual morphology including topological holes, hence termed "toric" spines $(n=76)$. More significantly, presynaptic terminals converging onto individual toric spines displayed numerous active zones (up to 49) derived from multiple axons (up to 11) with incoming trajectories distributed widely throughout 3D space. This arrangement is suited to integrate input sources. Dense reconstruction of two toric spines revealed that they were unconnected with the majority $(\sim 84 \%)$ of intertwined axons, implying a high capacity for information storage. We developed an ex vivo slice preparation and provide the first published data on space-specific neuron intrinsic properties, including cellular subtypes with and without toric-like spines. We propose that toric spines are a cellular locus of sensory integration and behavioral learning.
\end{abstract}

Key words: auditory processing; connectomics; dendritic spine; integration; neural circuit; synapse

\section{Significance Statement}

The majority of excitatory synapses in the brain are formed onto dendritic spines, which typically act to isolate the action of individual synapses. We discovered a new type of spine which in contrast receives convergent input from many different axons. These "toric" spines, named for their topological holes, are found on space-specific neurons in the barn owl auditory system. We used a combination of in vivo electrophysiology, super-resolution optical microscopy and serial block-face scanning electron microscopy (SBEM) to characterize the ultrastructure and wiring of toric spines, and ex vivo patch clamp recording to elucidate the cells' electrical properties. These data lead us to propose that toric spines serve as microanatomical hubs for neuronal computation, plasticity and learning.

\section{Introduction}

Neuronal computations are shaped by postsynaptic morphology (Rall, 1974). Across circuits and species, dendritic spines receive the majority of excitatory synaptic

Received May 24, 2019; accepted November 2, 2019; First published December 10, 2019.

The authors declare no competing financial interests.

Author contributions: D.S., E.A.B., M.C., D.F., and W.M.D. performed research; D.S., K.P., E.A.B., M.C., M.S., V.P., C.Z., E.H., A.S., N.M., P.L., D.F., and W.M.D. analyzed data; K.P., E.G.A., and W.M.D. contributed unpublished reagents/analytic tools; E.A.B., D.F., M.E., and W.M.D. designed research; W.M.D. wrote the paper.

This work was supported by National Institutes of Health Grants R01DC05640 (from NIDCD; to W.M.D.), BRAIN-STIM grant from the University input (Hering and Sheng, 2001). Most spines have narrow necks that serve to compartmentalize, electrically (Yuste, 2013; Kwon et al., 2017) and biochemically (Müller and Connor, 1991; Sabatini et al., 2001), a small number of

of California Davis Office of Research and Behavioral Health Center for Excellence (W.M.D.), R01NS104911 (from the BRAIN Initiative; to W.M.D. and M.H.E.), P41GM103412 (for support of National Center for Molecular Imaging Research; to M.H.E.), 2018 NARSAD Young Investigator grant (E.G.A.), NSF1754831 and MH114178 (to D.F.), and a UCDMC Interdepartmental Grant (D.F. and W.M.D.).

${ }^{*}$ D.S. and K.E.P. contributed equally to this work.

Acknowledgements: We thank Dr. Ingrid Brust-Mascher, Technical Director of the University of California Davis Health Sciences District Advanced Imaging Facility, for expert assistance with STED Imaging and Dr. Colin Reardon, 
synaptic inputs - usually one. In this arrangement the dendritic branch performs the first stage of integration of inputs coming from multiple sources (Poirazi et al., 2003). Here, we identify an alternative motif involving first stage integration via a new type of dendritic spine.

Space-specific neurons in the barn owl inferior colliculus (Knudsen and Konishi, 1978) compute sound source direction via integration of binaural cues, interaural time difference (ITD) and interaural level difference (ILD), and the elimination of phase ambiguity by convergence across frequency channels (Wagner et al., 1987; Olsen et al., 1989; Brainard et al., 1992; Konishi, 2003; Singheiser et al., 2012), computations known to involve multiplication and non-linear thresholding, respectively (Peña and Konishi, 2000, 2001, 2002, 2004). In addition, the responses of space-specific neurons can be modulated by visual input (Bergan and Knudsen, 2009) and attention (Winkowski and Knudsen, 2006; Mysore and Knudsen, 2014). Thus, space-specific neurons act as pattern detectors via the integration of complex sensory inputs, and make response adjustments based on behavioral context.

Space-specific neurons provide an opportunity to understand how neuronal computations are shaped by microanatomical patterns of synaptic convergence, but their ultrastructure is unknown. To fill this gap we used serial block-face scanning electron microscopy (SBEM; Denk and Horstmann, 2004) and stimulated emission depletion (STED) microscopy (Hell, 2003). Space-specific neurons in young adult owls were labeled in vivo by focal injection of retrograde tracer at functionally defined map locations (Rodriguez-Contreras et al., 2005). STED imaging revealed two cellular subtypes, one studded with typical spines and the other with a previously unreported type of spine with unusually complex and variable morphology, termed "toric" spines. SBEM imaging was used to reconstruct all 76 toric spines found on the soma and proximal dendrites of one space-specific neuron, along with their associated presynaptic terminals. In length, cytoplasmic volume and innervation density, toric spines resemble thorny excrescences found on pyramidal cells in mammalian hippocampus and amygdala (Amaral and Dent, 1981). Thorny excrescences receive numerous active zones (Chicurel and Harris, 1992) coming from one or at most two input sources (Wilke et al., 2013; e.g., individual mossy fibers in the hippocampus), forming the so-called detonator synapse (Urban et al., 2001). We found that toric spines receive a comparable number of active zones, but in contrast, these come from up to 11 different inputs. The latter arrangement is suited for integration, not detonation nor compartmentalization.

Faculty Director, and Dr. Douglas Rowland, Co-director of the University of California Davis Animal Imaging Shared Resource, for providing the MRI image of an owl brain.

Correspondence should be addressed to William M. DeBello at wmdebello@ucdavis.edu.

https://doi.org/10.1523/ENEURO.0197-19.2019

Copyright (C) 2020 Sanculi et al.

This is an open-access article distributed under the terms of the Creative Commons Attribution 4.0 International license, which permits unrestricted use, distribution and reproduction in any medium provided that the original work is properly attributed.
To date, knowledge of space-specific neuron function derives from extracellular recordings (numerous studies) and three reports using intracellular sharp electrodes (Peña and Konishi, 2001, 2002, 2004). Neither technique provides information on intrinsic electrical properties or can resolve individual synaptic events. We performed ex vivo patch-clamp recordings from putative space-specific neurons in brain slices from juvenile owls. Many of these cells exhibited large, atypical spines including toric-like structures, the apparent developmental precursors to mature toric spines. In total, these findings and newly established methods provide a path to investigate the causal connections between microanatomical structure and the neuronal computations that underlie high-level pattern detection.

\section{Materials and Methods}

\section{Animals}

A total of 12 barn owls (Tyto alba) of either sex were used in this study. Adult animals ( $>250 \mathrm{~d}$ old) were grouphoused in large flight aviaries while juveniles (35-40 d) remained in isolated nest boxes with parents and siblings. All husbandry and experimental methods were approved by the University of California Davis Institutional Animal Care and Use Committee.

\section{Microelectrode recording and retrograde labeling in vivo}

Four adult owls were used for in vivo retrograde labeling. Owls were anesthetized using $2 \%$ isofluorane, a mixture of nitrous oxide and oxygen (1:1) and wrapped in soft cloth. Craniotomies were opened above the tectal lobes and the owl was secured in a stereotax located inside a soundproof recording room. Insulated tungsten recording electrodes $\sim 6-\mathrm{M} \Omega$ impedance $(\mathrm{FHC})$ were lowered through the forebrain to the optic tectum (OT) based initially on stereotaxic coordinates $(17 \mathrm{~mm}$ rostral to the neck muscle insertion, $6 \mathrm{~mm}$ lateral to the midline) and then refined on the basis of response criteria. Visual receptive fields were measured by computer-controlled projection of static or moving dots onto a calibrated screen and the responses were used to navigate to the deep layers of OT (dOT; in 5/6 injections) based on characteristic latencies, preference for negative contrast stimuli, and absence of bursting behavior. Sites representing frontal space near $0^{\circ}$ azimuth were targeted. ITD tuning was determined via dichotic stimulation delivered through speakers (ED-21913-000, Knowles Electronics) positioned $5 \mathrm{~mm}$ from the tympanic membrane. Tungsten electrodes were removed and replaced with glass electrodes in the same location $(1.5 \mathrm{~mm}$ borosilicate glass, 10-20 $\mu \mathrm{m}$ tip), containing $10 \%$ biotinylated dextranconjugated tetramethylrhodamine $3000 \mathrm{MW}$ (microruby, \#D-7162; Thermo Fisher) or $10 \%$ lysine fixable dextran 3000 MW (Texas-red, \#D3328; Thermo Fisher) in 1\% potassium chloride solution. Chloridized silver wires were used to record physiologic responses to confirm injection location. lonophoretic injections were performed using 7-s on/off cycles of $3 \mu \mathrm{A}$ for $15 \mathrm{~min}$. Glass electrodes were withdrawn and the procedure was repeated on the 
Table 1. Owls

\begin{tabular}{|c|c|c|c|c|c|}
\hline \multicolumn{6}{|c|}{ Adult owls (>250 d old) } \\
\hline & \multicolumn{3}{|c|}{ Injection } & \multirow{2}{*}{$\begin{array}{c}\text { Imaged } \\
\text { cells }\end{array}$} & \multirow{2}{*}{$\begin{array}{l}\text { Imaging } \\
\text { method }\end{array}$} \\
\hline Owl & Side & site & Tracer & & \\
\hline 246 & L & Deep OT & Microruby & 1 & SBEM \\
\hline 213 & $\mathrm{R}$ & Deep OT & Microruby & 6 & STED \\
\hline 213 & L & Deep OT & Microruby & 8 & STED \\
\hline 229 & $\mathrm{R}$ & Deep OT & Microruby & 8 & STED \\
\hline 972 & $\mathrm{R}$ & $\operatorname{ICX}$ & Microruby & 11 & STED \\
\hline \multirow[t]{3}{*}{972} & L & Deep OT & Texas Red & 7 & STED \\
\hline & & & & 40 & \\
\hline & \multicolumn{5}{|c|}{ Juvenile owls } \\
\hline Owl & Purpose & Age (d) & $\begin{array}{l}\text { Patched } \\
\text { cells }\end{array}$ & $\begin{array}{l}\text { Imaged } \\
\text { cells }\end{array}$ & \\
\hline 2 & Intrinsic prop. & $35-40$ & 4 & 3 & \\
\hline 3 & Intrinsic prop. & $35-40$ & 5 & 3 & \\
\hline 4 & Intrinsic prop. & $35-40$ & 5 & 7 & \\
\hline 5 & Intrinsic prop. & $35-40$ & 4 & 1 & \\
\hline 6 & Intrinsic prop. & $35-40$ & 2 & 2 & \\
\hline 7 & Synaptic stim. & $40-50$ & 2 & 0 & \\
\hline \multirow[t]{2}{*}{8} & Synaptic stim. & $40-50$ & 1 & 0 & \\
\hline & & & 23 & 16 & \\
\hline
\end{tabular}

Owls and procedures.

opposite tectal lobe. In 1/6 injections, the external nucleus of the inferior colliculus (ICX) was directly targeted (Table 1). Following injections, craniotomies were disinfected with $1 \%$ chloroptic ointment. After 3 - to 7 -d survival time, owls were anesthetized using $5 \%$ isofluorane + 1:1 nitrous oxide/oxygen. Heparin (300 U) was injected into the left cardiac ventricle and the owl was perfused with $0.1 \mathrm{M}$ phosphate buffer ( $\mathrm{PO} 4, \mathrm{pH} 7.4)$, followed by $4 \%$ paraformaldehyde in PO4 buffer (for STED imaging; see below for tissue preparation for SBEM imaging). Brains were removed and placed in $30 \%$ sucrose and 0.1 M PB for $24 \mathrm{~h}$. Tectal lobes were isolated, sectioned at 50 $\mu \mathrm{m}$ on a vibratome (Leica), and mounted with an antifade agent (Prolong gold, P36930; Thermo Fisher).

\section{STED imaging and morphometric analysis}

Imaging was performed on a Leica TCS SP8 STED $3 \times$ confocal microscope (Leica Microsystems), using a $100 \times$ oil-immersion objective (HC PL APO CS2, 1.4 NA; Leica Microsystems). Parameters for STED imaging were white light laser, line $551 \mathrm{~nm}$ for stimulating, and 660-nm depletion laser, both between $20 \%$ and $40 \%$. Image frame size was $4800 \times 4800$. Step size for $z$-stacks was 17.44 $\mu \mathrm{m}$, resulting in pixel/voxel dimensions of $0.024,0.024$, and $0.18 \mu \mathrm{m}(x, y$, and $z$, respectively). Huygens Professional SVI software was used for deconvolution of all images, using a theoretical point spread function (PSF). All well-labeled neurons in the rostrolateral aspects of IC were imaged ( $n=39$ cells in perfusion-fixed tissue from three adult owls; $n=16$ cells in immersion-fixed tissue from five juvenile owls as described later).

STED images were analyzed using Imaris 8.1 (Bitplane $A G)$. Surface creation was used to measure surface area and volume of the soma and primary dendrites. To isolate the primary dendrite, the slice tool was used to separate the base of the dendrite from the soma at the point where the dendritic caliber first became uniform (i.e., avoiding sharp curvature at the interface). The cross-sectional surface of the ensuing dendritic face was used to measure thickness of primary dendrites.

Cell classification was accomplished using Imaris' 3D view. For adult tissue, cells containing toric spines were classified as Type I, while cells lacking toric spines were classified as Type II. For juvenile tissue the cell population was more heterogeneous. Cells were not classified as Type I or Type II but scored for the presence or absence of toric-like spines.

\section{SBEM}

In one owl, microelectrode recordings were made as described above followed by multiple tracer injections in deep OT to increase the likelihood of generating a completely filled neuron. The owl was perfused as before but with $1 \%$ glutaraldehyde in cacodylate buffer (Wilke et al., 2013); $150-\mu \mathrm{m}$ vibratome sections were collected and shipped to University of California San Diego for further processing and imaging. Sections were freeze-fractured, reacted en bloc with streptavidin-HRP (which binds to the biotin moiety on microruby) and detected using diaminobenzidine (DAB). Light microscope image (LM) of the section at this point was obtained. Following osmication and staining with heavy metals the tissue was extremely opaque to visible light, obscuring internal structure. To target the labeled cell for SBEM volume imaging, a Zeiss Versa $510 \mathrm{x}$-ray microscope (XRM) was used to generate $3 \mathrm{D}$ tomographic volumes relative to tissue landmarks with $0.7-\mu \mathrm{m}$ pixel resolution. Based on the XRM volume, a 45 $\times 75 \times 75 \mu \mathrm{m}$ tissue block centered on the labeled cell was targeted and imaged with a Gatan 3View unit on a Zeiss Merlin SEM at $2.5 \mathrm{kV}$, with a magnification of $2800 \times, 15 \times 15 \mathrm{k}$ raster size, and $1-\mu$ s pixel dwell time. The final pixel size was $5 \mathrm{~nm}$ and the $Z$ step size was 70 $\mathrm{nm}$. The imaging parameters yield sufficient resolution to identify synaptic contacts while maximizing the rate of data collection. The SBEM image volume was postprocessed for proper alignment at University of California, San Diego and annotated at University of California, Davis.

\section{SBEM analysis}

A team of eight annotators used the open source platform IMOD (https://bio3d.colorado.edu/imod/) to analyze the SBEM image volume. It contained 54 cell nuclei representing six distinct cell types (neuronal and glial somatic profiles), four putative space-specific neurons, one of which was well labeled throughout entire cytosolic compartment including spines, an estimated 100s of myelinated axons, 1000 s of unmyelinated axons and 10,000s of synapses. The labeled space-specific neuron was traced through all sections, meshed and rendered in 3D. This reconstruction includes the entire soma and proximal dendrites but not distal dendrites as they extended outside of the volume (space-specific neuron dendritic fields can be $\sim 200 \mu \mathrm{m}$ in diameter). Each toric spine was numbered and isolated for further analysis. The surface areas and contour volumes were determined using the imodinfo command, which calculates surface area by adding the areas of the triangles making up the mesh and the volume by summing 
the areas of each contour times the $Z$ distance connecting the contours. The number of holes was assessed by visual inspection of the 3D model. Because the cytosolic label was dense, in most cases it was not clear whether these tori were cytoplasmically contiguous or interrupted by tight junctions, although one clear example of each was found.

Twenty-seven toric spines representing the full spectrum of complexities were selected for further analysis. All unlabeled axons innervating these 27 spines were traced outwards from their points of synaptic contact until the axonal process became ambiguous or reached the end of the volume.

\section{Synapse identification in SBEM}

Synapses were identified by the presence and distribution of presynaptic vesicles. Active zones were identified using the following criteria: (1) a cluster of five or more presynaptic vesicles, (2) vesicle cluster apposed to the presynaptic membrane, (3) parallel presynaptic and postsynaptic membranes (no gaps), (4) these features present in at least two consecutive sections. In some cases, the postsynaptic density (PSD) could be discerned but the presence of the dark $\mathrm{DAB}$ label made this an unworkable requirement.

\section{Axonal trajectory analysis}

Seven spines whose axons had been extended for longer distances without ambiguity were selected for trajectory analysis. The length, number of boutons and outgoing trajectories at each axonal end were measured. Length was measured by manual skeletonization. Boutons were defined as axonal swellings containing presynaptic vesicles. Trajectories were determined by marking the $x y z$-coordinates of the skeleton endpoint as well as a second internal skeletal point within $2 \mu \mathrm{m}$ of the skeleton endpoint. A small number of axons branched and were split into segments. If the axon had a clear main segment with a smaller side branch it was classified as a "T" branch and the axon was split into two segments; the main segment and side branch. The length and number of boutons were measured separately for each segment. For trajectory analysis, only the two ends of the main segment of these axons were included. If the axon branched into two pieces that were comparable and a "main segment" could not be distinguished, it was classified as a "Y"branch and split into three segments at the branch point. End 1 of each segment was defined as the open end, and end 2 was the branch point. Only one analyzed axon was classified as a Y-branch. For this axon, the trajectory was measured at all three open ends.

Axonal trajectories were analyzed by custom scripts written in MATLAB (MathWorks). The two points at each end were converted to a $3 D$ vector with the outside point as the vector head and the inside point as the vector tail. All $z$-coordinates were scaled by a $z$ ratio of 13.78 pixels in $x-y / z$ section. Each end vector was scaled to a length of 1 and plotted in 3D with its tail at the origin. End vectors were grouped together by spine, with the two vectors from the same axon paired together. To determine whether input trajectories were clustered, the distance between vectors was measured as the minimum angle of arc drawn from the head of one vector to the head of the other. Vector distance $(\theta)$ was calculated from the dot product of the Euclidean vectors:

$$
\cos \varnothing=\frac{\vec{u} \cdot \vec{v}}{\vec{u}^{*} \vec{v}}
$$

Distances were calculated pairwise between each end vector and every other end vector on the same spine, except the other end of the same axon. The distance between the two end vectors from the same axon was called "angle of separation" to differentiate from other vector distances. This is a proxy measure for curvature of the axon. A perfectly straight axon yields an angle of separation of $180^{\circ}$. The single Y-branched axon on TS24 was included in the vector plots but excluded from the distance calculations for simplicity.

\section{Connection fraction (CF)}

Potential connectivity for TS1 and TS7 was assessed by partial reconstruction of all unmyelinated axons that passed within $2 \mu \mathrm{m}$ of the spine. CF was calculated as (the number of axons that synapse with the spine/the total number of axons within $2 \mu \mathrm{m})$. To determine the probability envelope of observing the actual distribution of active zones across each potential axonal input, a bootstrap analysis was constructed based on the simple assumption of equal access. The simulation was run 10,000 times for each spine to determine mean values and \pm 2 SD.

\section{In vitro slice preparation}

Six juvenile owls (35-40 d old) were used for current clamp experiments to determine intrinsic properties and two juveniles (40-50 d old) were used for voltage-clamp experiments to measure EPSCs. Juvenile owls were anesthetized with isoflurane and perfused transcardially with heparin-containing (300 units/l) artificial CSF (aCSF; $124 \mathrm{mM}$ $\mathrm{NaCl}, 1.3 \mathrm{mM} \mathrm{KCl}, 1 \mathrm{mM} \mathrm{NaH} \mathrm{PO}_{4}, 26.2 \mathrm{mM} \mathrm{NaHCO}_{3}, 1.3$ $\mathrm{mM} \mathrm{MgCl} 2,2.5 \mathrm{mM} \mathrm{CaCl}_{2}$, and $11 \mathrm{mM}$ glucose). Brains were rapidly removed, blocked, and placed in ice-cold modified aCSF (248 mM sucrose, $11 \mathrm{mM}$ glucose, $1.3 \mathrm{mM}$ $\mathrm{KCl}, 1 \mathrm{mM} \mathrm{NaH}_{2} \mathrm{PO}_{4}, 26.2 \mathrm{mM} \mathrm{NaHCO}_{3}, 1.3 \mathrm{mM} \mathrm{MgCl}_{2}$, and $2.5 \mathrm{mM} \mathrm{CaCl}_{2}$ ). Horizontal midbrain sections $(250$ $\mu \mathrm{M})$ containing ICX and lateral shell of the central nucleus of the inferior colliculus (ICCls) were cut on a vibratome (Leica VT1200S) and transferred to an incubation chamber containing aCSF at $32^{\circ} \mathrm{C}$ for $30 \mathrm{~min}$ before moving to aCSF at room temperature until used for recordings. Recordings were made in a submersion chamber perfused with aCSF $(2 \mathrm{ml} / \mathrm{min})$ at room temperature. All solutions were bubbled with $95 \% \mathrm{O}_{2}-5 \%$ $\mathrm{CO}_{2}$ continuously.

\section{Patch-clamp recordings}

Whole-cell patch-clamp recordings were made from visually identified cells in the ICX region using borosilicate glass pipettes (2-4 M $\Omega$ ). For current-clamp experiments, pipettes were filled with $\mathrm{K}$-methanesulfonate-based internal solution (135 mM CH3KO3S, 10 mM HEPES, 1 mM 


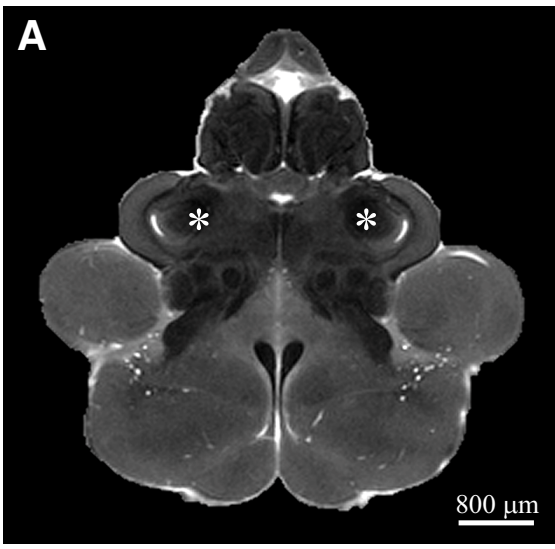

D

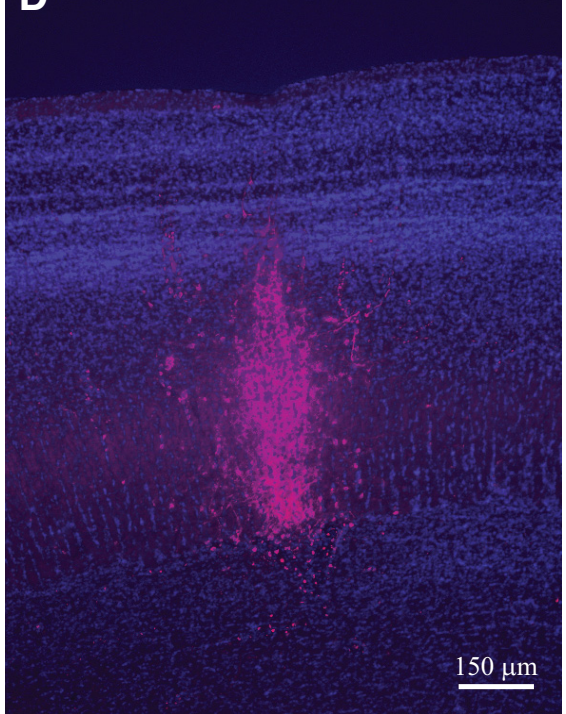

B
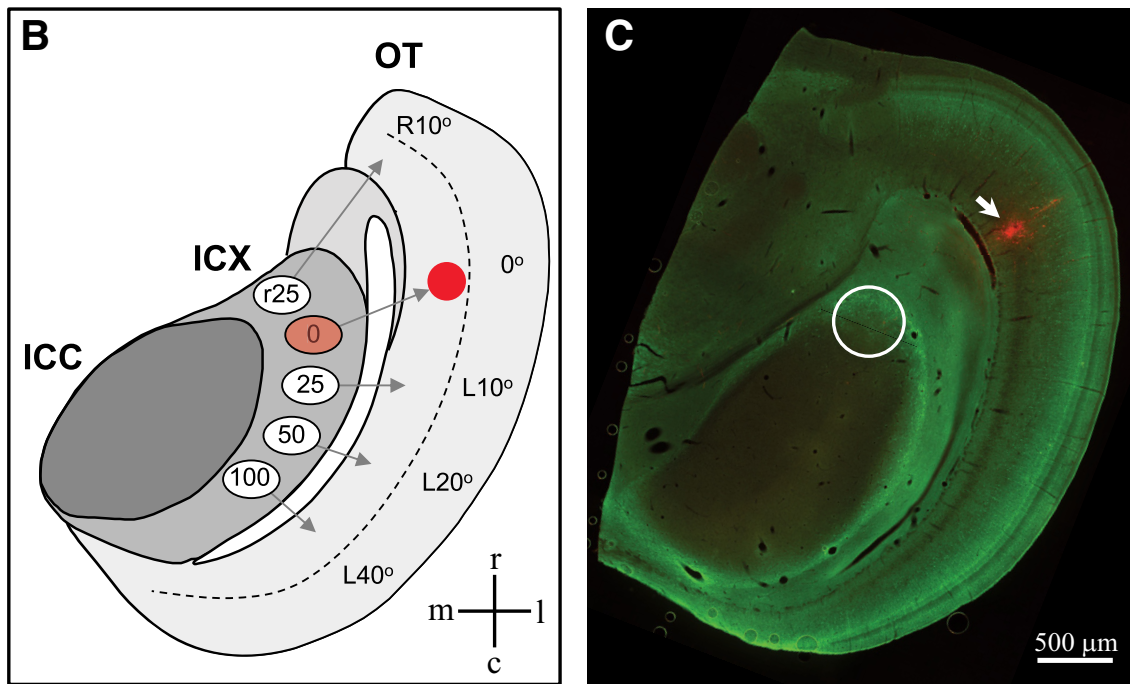

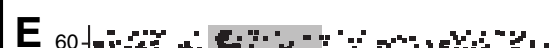

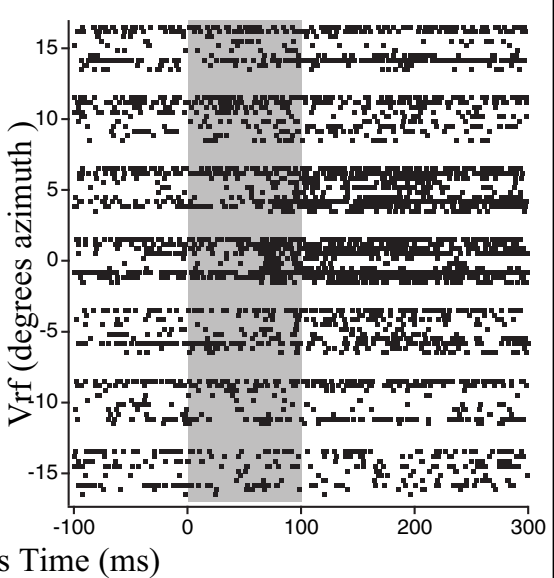
E $60-4$ and

$40-y^{2}$ 7)

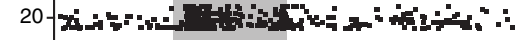
(2) "y 可 0 and An 是

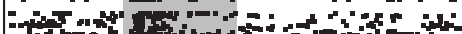

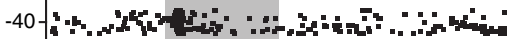

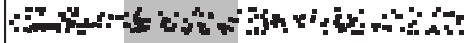

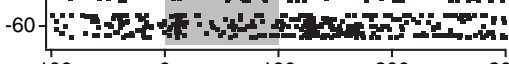

Peristimulus Time (ms)

Figure 1. In vivo labeling of SSNs. A, MRI image of owl brain with tectal lobes indicated by asterisks. B, Diagram of horizontal section through R tectal lobe. ITD tuning of SSNs in ICX is indicated in microseconds left-ear leading. Visual receptive field (Vrf) location is indicated in degrees azimuth SSNs project to the deep layers of the OT where the auditory map aligns and integrates with visual space map indicated in degrees azimuth. Tracer injection at $0 \mu \mathrm{s} / 0^{\circ}$ in the dOT (solid red) retrogradely labels SSNs at the cognate map locations in the inferior colliculus. C, Representative horizontal section (50 $\mu \mathrm{m}$ thick) shows location of injection site (arrow) and location of retrogradely labeled neurons in the inferior colliculus (circle). Green label is immunostaining for CaMKII which is highly expressed in the lateral aspects of ICX. The border between the ICCls and ICX is not well delineated. D, Higher magnification view of a representative injection (red) in the dOT. Blue label is DAPI staining. $\boldsymbol{E}$, Raster plots displaying the auditory (left) and visual (right) tuning recorded at the site of injection. IC, inferior colliculus; ICCls, lateral shell of the central nucleus of the inferior colliculus; ICX, external nucleus of the inferior colliculus; SSN, space-specific neuron.

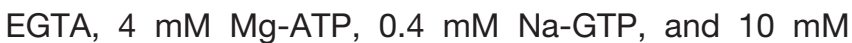
phosphocreatine; $310 \mathrm{mOsm}$ ) containing $0.5 \%$ biocytin (Thermo Fisher). For voltage-clamp experiments, a Csmethanesulfonate-based internal (120 $\mathrm{mM} \mathrm{CH}_{3} \mathrm{CsO}_{3} \mathrm{~S}, 15$ mM CsCl, 8 mM NaCl, 10 mM TEA-Cl, 10 mM HEPES, 0.5 mM EGTA, 2 mM QX314, $4 \mathrm{mM} \mathrm{Mg-ATP,} \mathrm{and} 0.3 \mathrm{mM}$ Na-GTP; $310 \mathrm{mOsm}$ ) was used. All chemicals were from Sigma. Recordings were acquired using a Multiclamp 700B amplifier (Molecular Devices), digitized at $20 \mathrm{kHz}$ with a Digidata 1550 digitizer (Molecular Devices), and low-pass filtered at $8 \mathrm{kHz}$. Evoked EPSCs were elicited in cells voltage-clamped at $-70 \mathrm{mV}$ in response to a brief electrical stimulus $(0.2 \mathrm{~ms}, 20-50 \mu \mathrm{A})$ delivered through a concentric bipolar stimulating electrode (FHC). The stimulating electrode was placed locally in the ICCls. Synaptic blockers (bicuculine: $20 \mu \mathrm{M}$; NBQX: $10 \mu \mathrm{M}$; both from Sigma) were washed in during recordings, as indicated. Slices were immersion-fixed in 4\% paraformaldehyde and stained with SA-488 for STED imaging.

\section{Data analysis}

Resting membrane potential (in $\mathrm{mV}$ ) was measured while injecting no current $(I=0)$ immediately after breaking into a cell (average of first 500 ms). Membrane capacitance was estimated using a single-exponential fit to the first 200 ms of the response to a 0.5-s injection of hyperpolarizing current. Membrane resistance was measured from the linear portion of a current clamp input-output curve as the slope of the line fitted to the voltages recorded at increasing current injections. Action potentials 
A

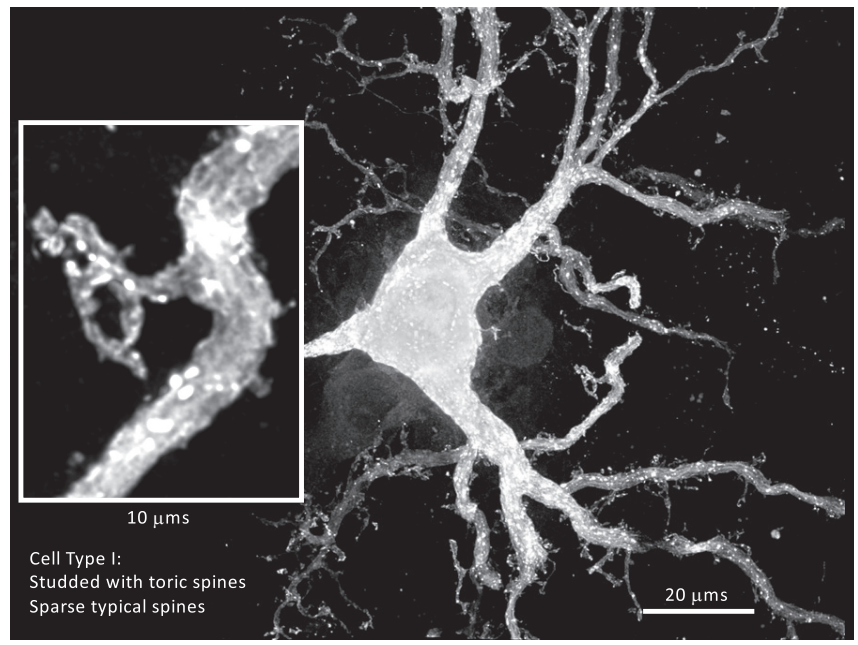

C

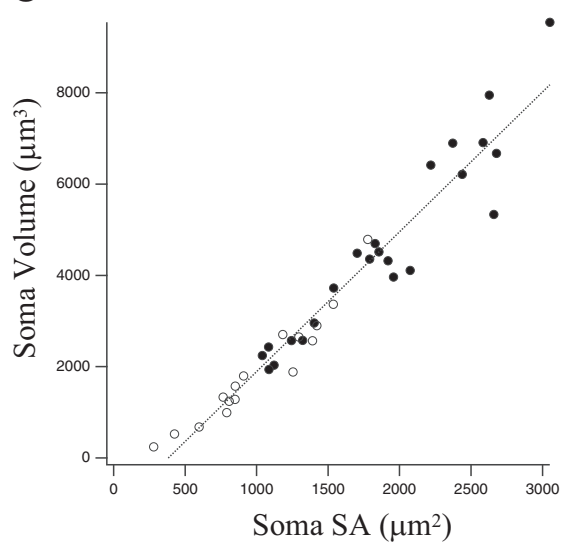

B

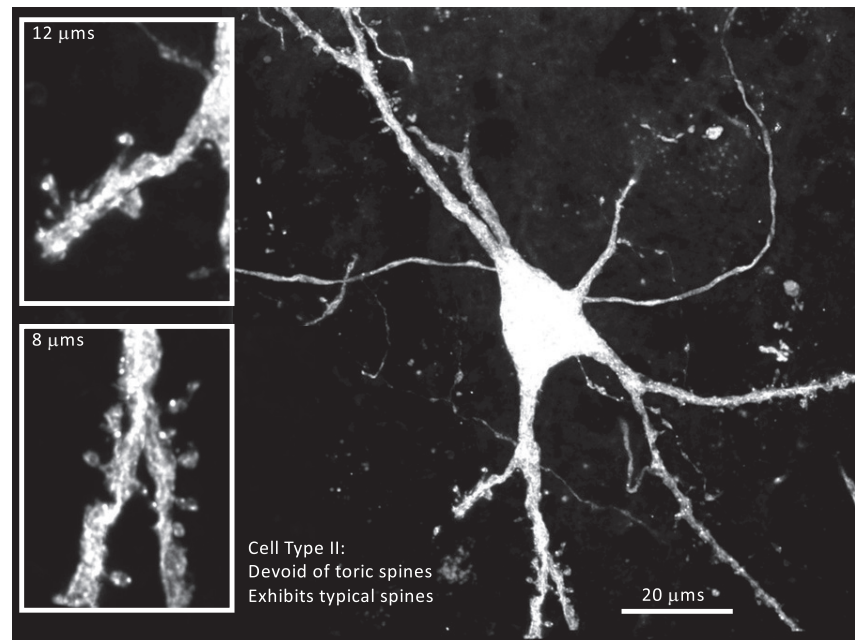

D

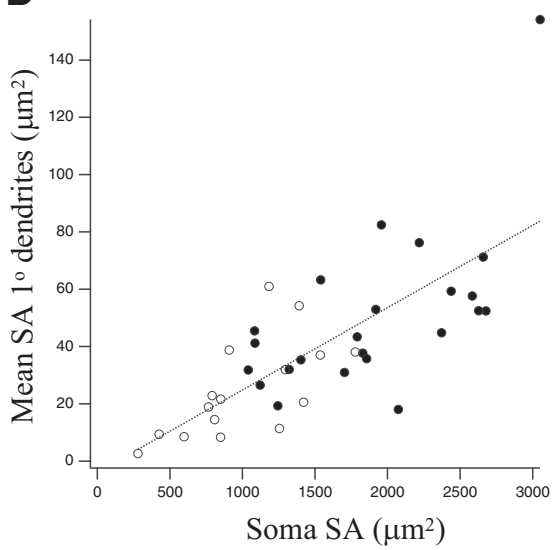

E

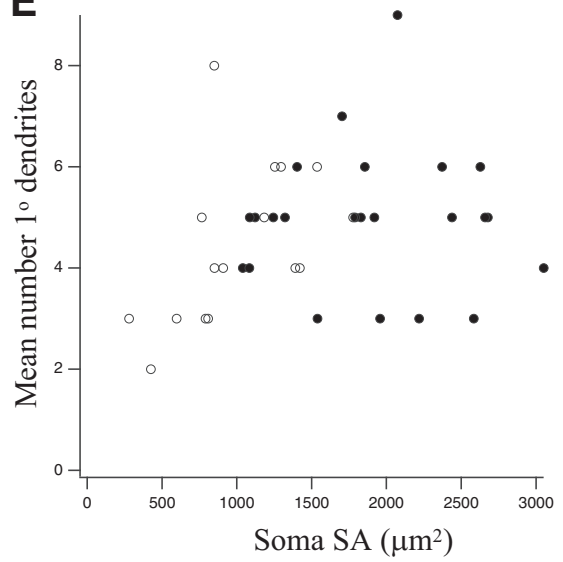

F

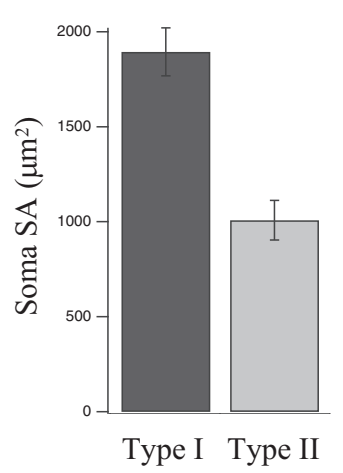

G

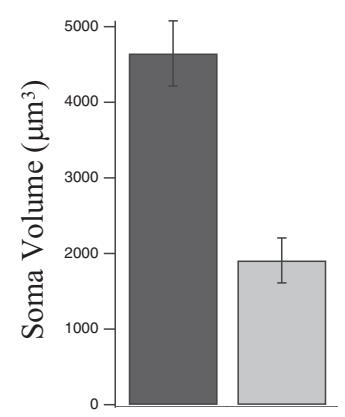

Type I Type II
H

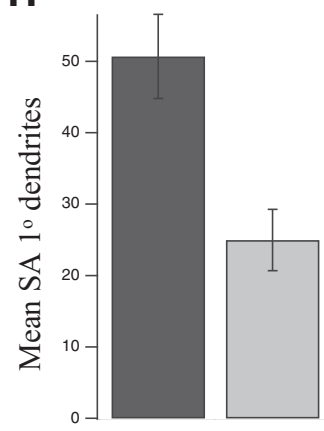

Type I Type II
I

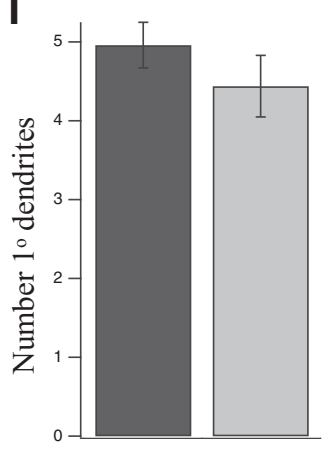

Type I Type II

Figure 2. Type I and Type II SSNs. STED images of representative examples of Type I $(\boldsymbol{A})$ and Type II (B) SSNs; $N=39$ SSNs total, 23 Type I (solid circles in $\boldsymbol{C}-\boldsymbol{E}$ ) and 16 Type II (open circles in $\boldsymbol{C}-\boldsymbol{E}$ ). $\boldsymbol{C}$, Soma volume versus surface area across both types: $r=0.968$, $r^{2}=0.936, p<0.00001$. $\boldsymbol{D}$, Mean cross sectional surface area of primary dendrites versus surface area of the soma: $r=0.728, r^{2}$ $=0.530, p<0.00001$. $\boldsymbol{E}$, Mean number of primary dendrites versus surface area of the soma: $r=0.243, r^{2}=0.059, p=0.1356$. $\boldsymbol{F}$, Mean somatic surface area (Type I $=1895 \mu \mathrm{m}^{2}$ versus Type II $\left.=1008 \mu \mathrm{m}^{2} ; p<0.00001\right)$. G, Mean somatic volume (Type I $=4648$ $\mu \mathrm{m}^{3}$ vs Type II = $\left.1909 \mu \mathrm{m}^{3} ; p=0.00006\right)$. $\boldsymbol{H}$, Mean cross sectional surface area of primary dendrites $\left(\right.$ Type I $=50.7 \mu \mathrm{m}^{2}$ vs Type $\|=25.0 \mu \mathrm{m}^{2} ; p=0.00148$ ). I, Mean number of primary dendrites (Type I = 4.96; Type II = 4.44; $p=0.271$ ). Comparisons for $\boldsymbol{F}$-I were based on Mann-Whitney $U$ test. Error bars are SEM. 
LM

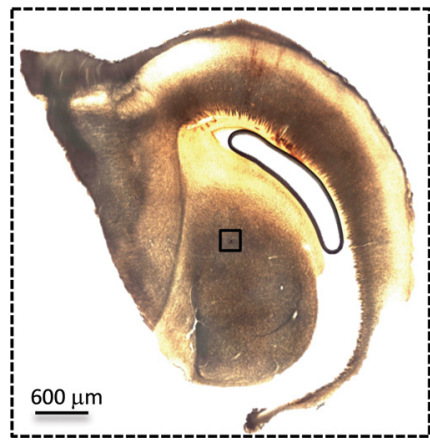

XRM

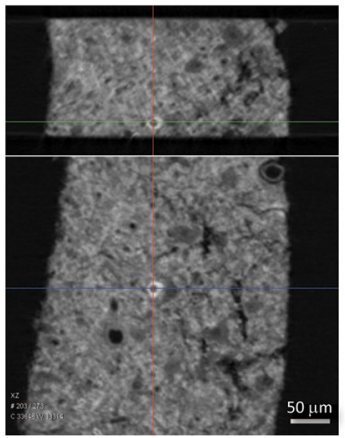

SBEM

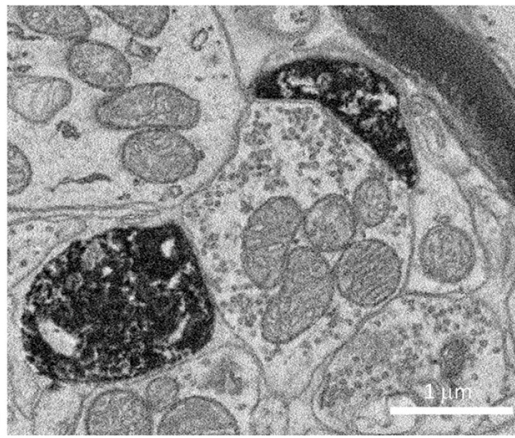

Figure 3. SBEM pipeline and SSN reconstruction. Light microscopy (LM) of 150- $\mu$ m-thick section reacted en bloc for DAB, before osmication. The labeled SSN is shown in the box and appears near the border of ICCls and ICX. XRM, X-ray microscopy was used to locate the SSN after osmication; SBEM was used to image a volume $75 \times 75 \times 45 \mu \mathrm{m}$ at 5-nm resolution.

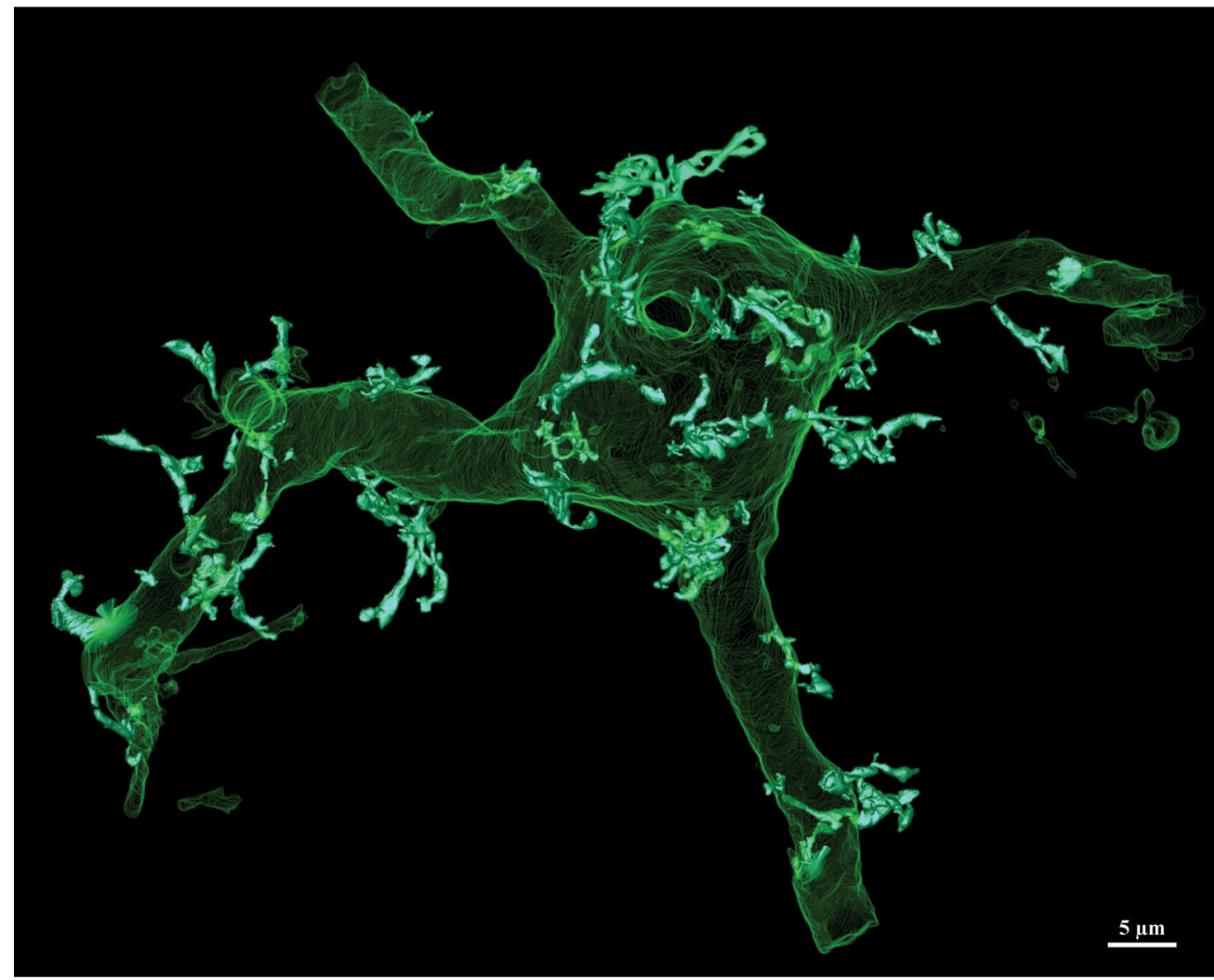

Figure 4. Reconstructed Type I SSN laden with toric spines. Full reconstruction of all labeled processes contained within the volume. Somatodendritic architecture is rendered in mesh green; toric spines in solid white.

were elicited by increasing depolarizing steps $(0.1-3 \mathrm{nA}$, $0.5 \mathrm{~s})$ delivered every $15 \mathrm{~s}$. Spikes were identified using a $20 \mathrm{mV} / \mathrm{ms}$ threshold of the first-order derivative of membrane potential. Results were identical when a more conservative $50 \mathrm{mV} / \mathrm{ms}$ detection threshold was applied. Spike onset was determined using the 2nd-order derivative (maximum acceleration) of membrane potential within a 2-ms time window before and after threshold crossing. Spike peak voltage $\left(V_{P}\right)$ was defined as the maximal $V$ within a 2-ms time window from threshold crossing and was used to calculate spike amplitude (peak V minus $V$ at spike onset). First spike latency was defined as the time between stimulus onset and first spike onset, and inter- spike interval as the time between consecutive spike onsets. All data were analyzed with custom scripts written in MATLAB.

\section{Results}

The strategy for labeling space-specific neurons is shown in Figure 1. Tungsten and glass electrodes were used to record multiunit activity in the tectal lobes, which include the inferior colliculus (IC) and OT. The lobes are shown in a magnetic resonance image of an intact owl brain at mid-transverse plane (Fig. 1A). Space-specific neurons found in the ICX are tuned for distinct values of ITD, as illustrated in Figure $1 B$. Their auditory spatial 


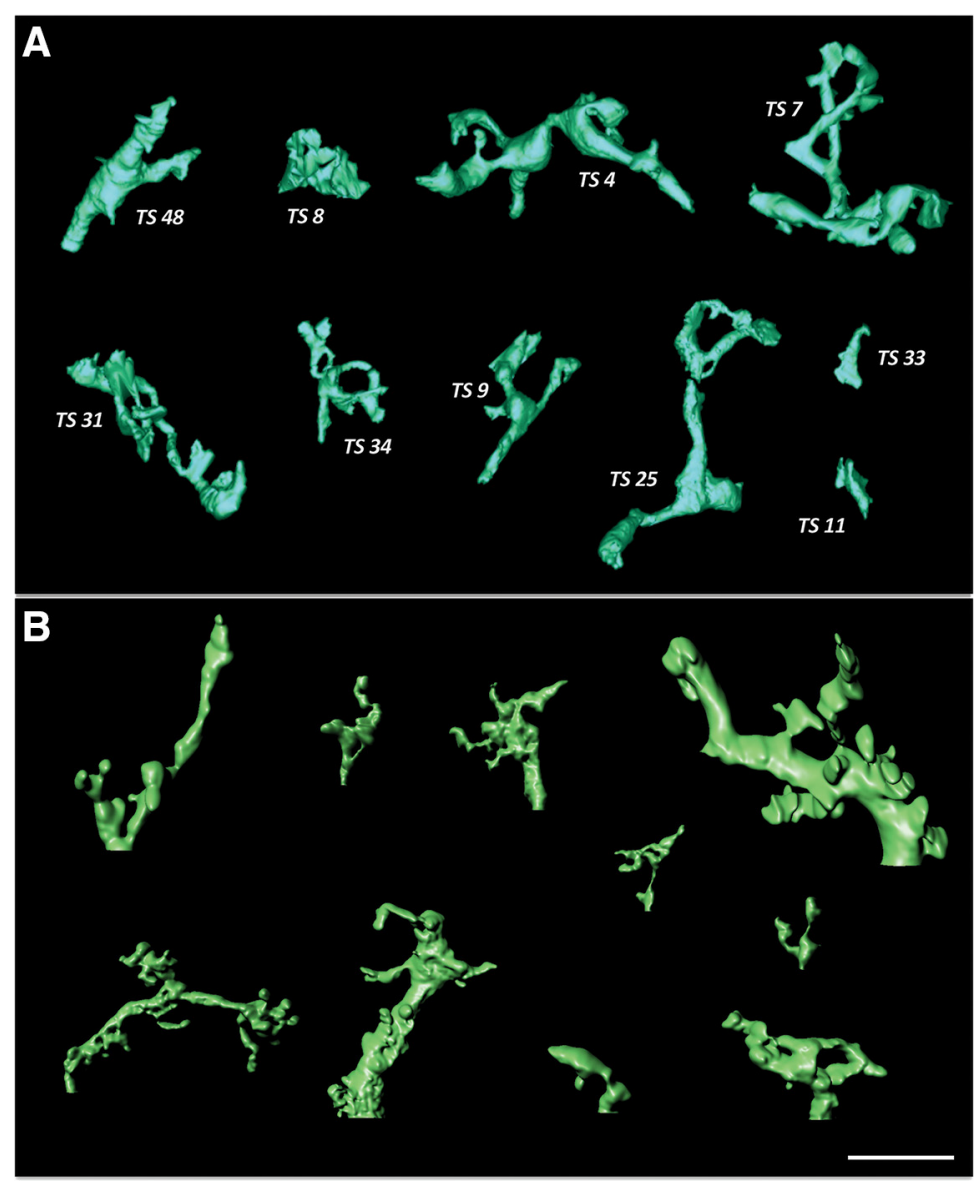

Figure 5. Toric spine zoo. A, SBEM-based reconstructions of 10 example toric spines, selected to cover the range of diversity in size, shape and complexity. Holes were present in $37 \%$ of spines (29/78 spines). Note the relatively uniform process diameter and relative lack of spine heads. $\boldsymbol{B}$, STED-based reconstructions of 10 example toric spines, selected as above. The apparent ultrathin features result from thresholding a locally variable concentration of intracellular tracer as opposed to volumetric reconstruction based on plasma membrane tracing in SBEM. Otherwise, the population of spine morphologies derived from each imaging method appear indistinguishable. Scale bar $=5 \mu \mathrm{m}$ (both panels).

receptive fields are constructed from convergent inputs which originate in the ICCls and other structures not shown (see Discussion). Space-specific neurons were labeled by injection of the tracer microruby or Texas-red in the dOT (5/6 injections), which receive monosynaptic input from space-specific neurons. Injection sites were confined to the deep layers with minimal rostrocaudal spread (Fig. 1C,D). ITD and visual spatial tuning at the injection sites (Fig. 1E) represented frontal space; as expected, the retrogradely labeled neurons were found within the region of the inferior colliculus representing frontal space, shown by the white circle in Figure $1 C$. This region includes the full mediolateral extent of ICX as well as lateral aspects of ICCls. Because no cytoarchitectonic marker that delineates the border between ICCls and ICX (Takahashi et al., 1987; Wagner et al., 2003) was compatible with the three tissue preparation methods used in this study (glutaraldehyde perfusion, $150-\mu \mathrm{m}$ section; paraformaldehyde perfusion, $50-\mu \mathrm{m}$ section; in vitro slice, $250-\mu \mathrm{m}$ section), neurons were not assigned to one or the other structure. In one case the injection was directly targeted to ICX; labeled neurons resulting from this injection were indistinguishable in both location and morphology from those labeled by the five dOT injections and therefore included in the analysis below. No neurons outside of the ICClsICX microcircuit were analyzed.

The tracer often produced complete cell-fills, revealing fine aspects of dendritic structure, similar to previous reports using in vivo labeling (Peña and Konishi, 2001) or immunostaining for CaMKII (Rodriguez-Contreras et al., 2005; Niederleitner and Luksch, 2012). From these studies, it was known that space-specific neurons are large multipolar cells with dendritic protrusions which often included typical spines. Unlike previous studies, we used high-resolution methods to reveal, for the first time, their ultrastructure. STED microscopy of 39 well-labeled space-specific neurons derived from five injections in three owls revealed two cell classes within a spectrum of overlapping morphologic features. The most striking difference was the presence of highly atypical dendritic spines on a subset of cells, defined here as Type I spacespecific neurons. These large structures exhibited tubular build, tended to lack spine heads and were often riddled with holes (Fig. 2A). The morphologic diversity of toric spines was so large that no single feature distinguishing them from typical spines was present in all. Type I space- 


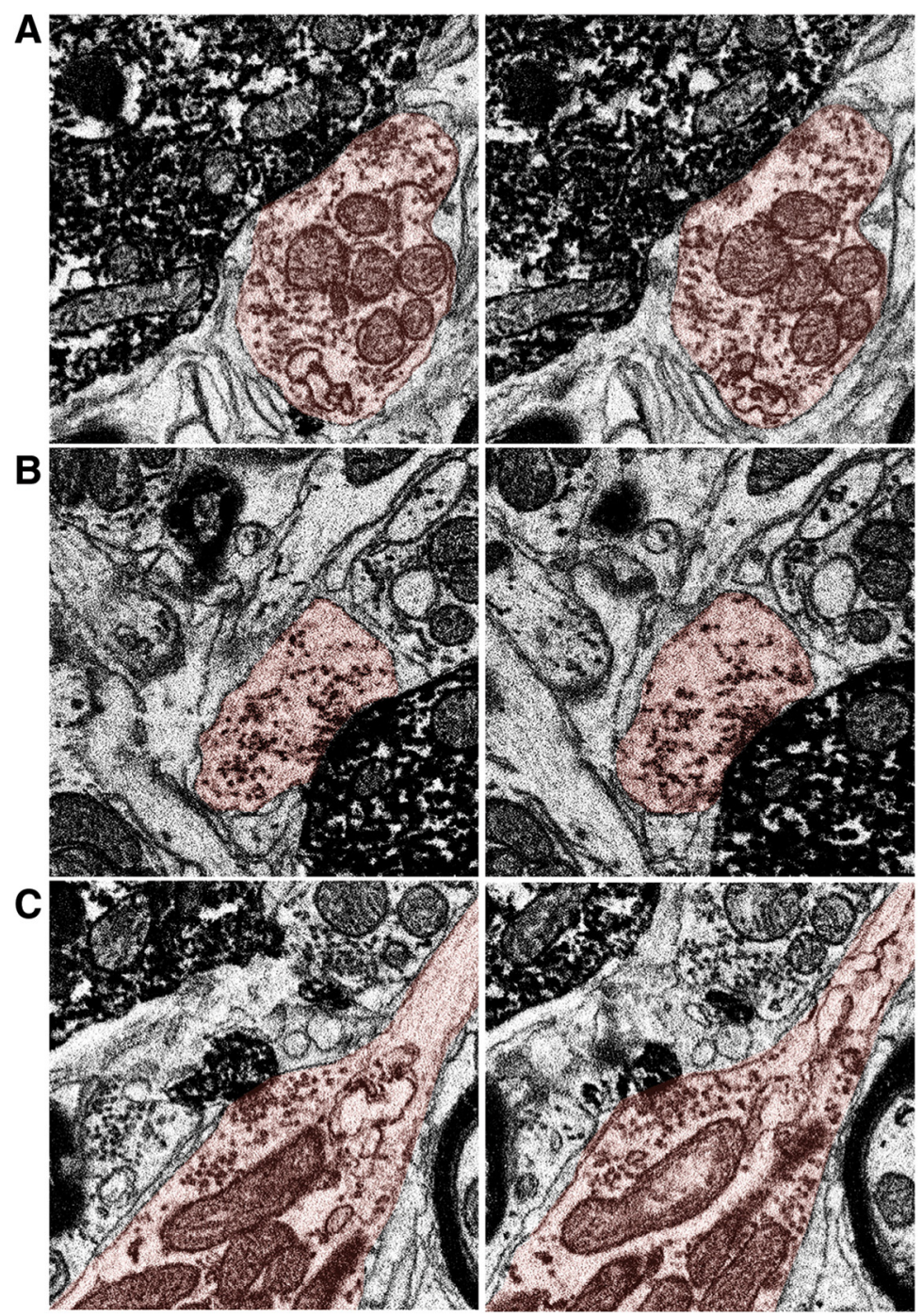

Figure 6. Synapse identification. Three representative synapses are shown onto the soma $(\boldsymbol{A})$, dendrite $(\boldsymbol{B})$, and toric spine $(\boldsymbol{C})$ of the labeled SSN. For each synapse two successive EM sections are shown. The SSN is darkly labeled and the presynaptic compartment has been traced (light orange fill). The synapse on the soma is bifurcated, with two separate active zones from the same bouton. Bifurcated synapses were also common on the dendrites and toric spines. The axon shown in the bottom images makes synapses onto TS1 (larger spine) and TS62 (smaller spine; synapse shown in EM images), as well as the soma (data not shown).

specific neurons were studded with toric spines and a paucity of typical spines, sometimes none (Fig. 2A). Type II space-specific neurons, defined here, were studded with a high density of typical spines and devoid of toric spines (Fig. 2B). In addition, Type I space-specific neurons tended to have larger somas (Fig. 2C,F,G) and thicker primary dendrites (Fig. 2D,H). The number of primary dendrites was not significantly different (Fig. 2E,I).

While the resolution of STED was sufficient to identify toric spines when they presented in the right orientation, high anisotropy ( $\sim 40 \mathrm{~nm}$ lateral, $\sim 600 \mathrm{~nm}$ axial resolution) introduced unacceptable distortions to the apparent 3D structure of spines presenting in other orientations (the majority of spines). Combined with high morphologic diversity and narrow tubular structure, volumetric measurement (toric vs typical) were not informative for the population of spines as a whole. We therefore turned to SBEM. Space-specific neurons were labeled as before, this time with multiple injections in the deep OT to increase the chance of detection, the brain perfused with glutaraldehyde and reacted en bloc with $\mathrm{DAB}$ to produce a durable reaction product that survived all subsequent processing. One well-labeled space-specific neuron was found on the ICCls-ICX border (Fig. 3, LM panel). After osmication the labeled space-specific neuron was isolated using x-ray microscopy (Fig. 3, XRM panel) and a $75 \times 75 \times 45 \mu \mathrm{m}$ volume $\left(253,125 \mu \mathrm{m}^{3}\right)$ imaged at $5 \mathrm{~nm}$ lateral resolution.

The DAB reaction product completely filled the neuron, which received profuse synaptic contacts from many unlabeled afferents (Fig. 3, SBEM panel). Volumetric reconstruction revealed a large multipolar neuron (Fig. 4) resembling the Type I space-specific neurons identified by STED. In particular, the soma and its six thick primary dendrites were studded with 76 toric spines (TS1 - TS76) and devoid of typical spines. Comparison of representative 

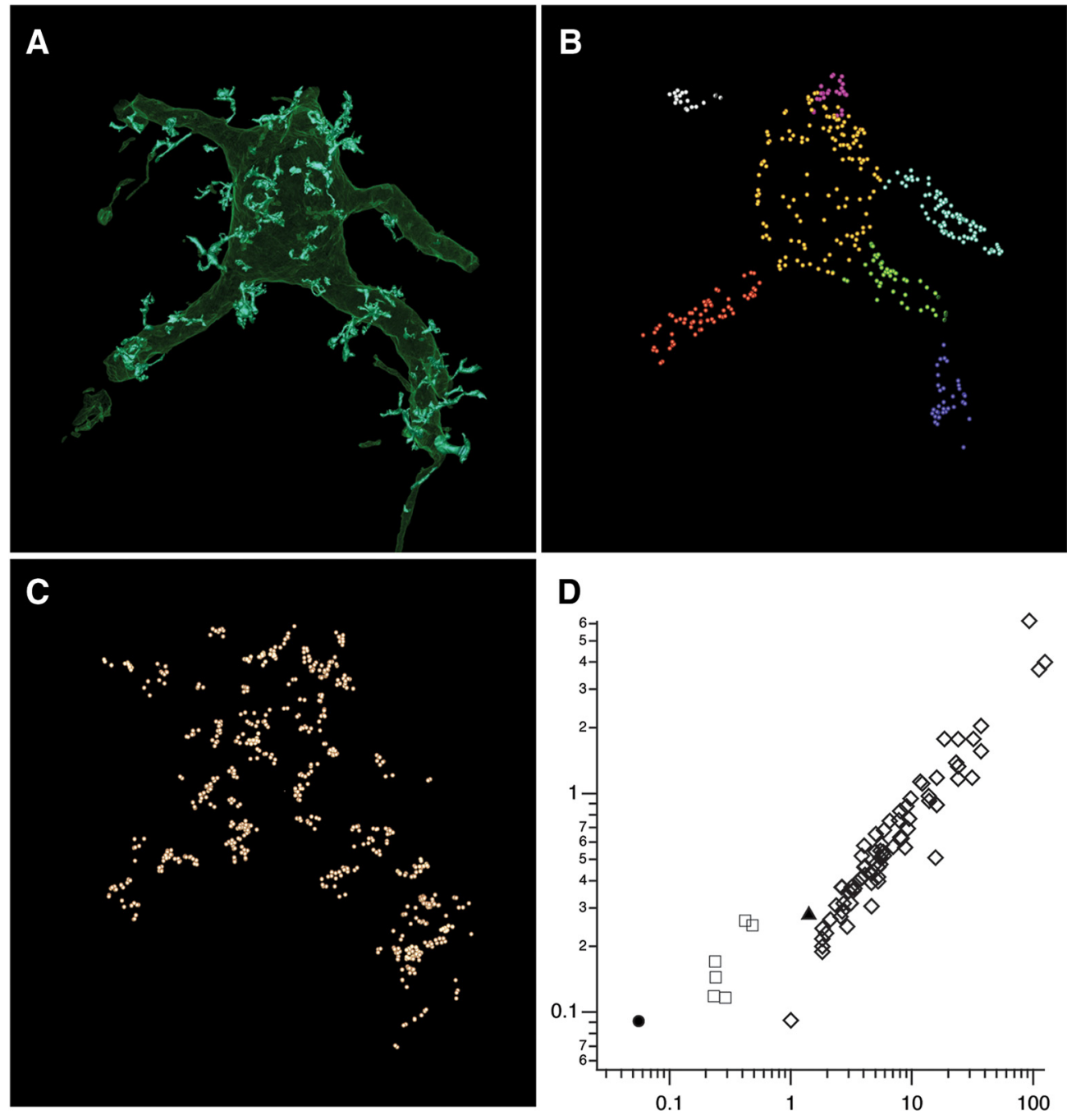

Figure 7. Distribution of synaptic input onto the labeled SSN. A team of annotators independently marked all active zones on the labeled SSN using criteria described in Materials and Methods. $\boldsymbol{A}$, Labeled SSN without active zones. Scale bar $=5 \mu \mathrm{m}(\boldsymbol{A}-\boldsymbol{C})$. $\boldsymbol{B}, \mathbf{A}$ total of 754 active zones was found on the soma (bright yellow; 228) and five primary dendrites (multiple colors; 526 total). $\mathbf{C}$, A total of 622 active zones were found on 76 toric spines (pale orange spheres). $\boldsymbol{D}$, Innervation density indicated by active zones per surface area $(y$-axis) or active zones per volume ( $x$-axis) was on average largest for toric spines (open diamonds; mean $=0.81$ and 12.6 for $\mathrm{AZ} / \mu \mathrm{m}^{2}$ and $\mathrm{AZ} / \mu \mathrm{m}^{3}$, respectively) than dendrites (open squares; mean $=0.177$ and 0.32 ), soma (filled circle; 0.091 and 0.056 ), or thin process (filled triangle; 0.279 and 1.41).

"zoos" of toric spines identified by SBEM or STED (Fig. 5) indicated that these populations are qualitatively indistinguishable. A thin process resembling an axon emerged from near the base of one primary dendrite. No somatic axon was found.

To map synaptic input, all active zones were identified by a team of annotators using criteria defined in Materials and Methods. Active zones occurring on the soma, dendrites and toric spines appeared similar as a relatively homogenous population of chemical synapses with clusters of synaptic vesicles apposed to the postsynaptic membrane (Fig. 6). Bifurcated synapses with two or more active zones belonging to the same axonal bouton were occasionally observed on all compartments. 622 active zones were found on toric spines and 754 on the soma and dendrites, indicating that toric spines are a major input hub (Fig. $7 A-C$ ). The innervation density (\# active zones per surface area) of toric spines was on average higher than that observed on the six primary dendrites, soma, or thin process (Fig. 7D).

Quantification of the ultrastructural features of toric spines is shown in Figure 8. Volumes (mean $=1.99 \times 10^{9}$ $\mathrm{nm}^{3}$ ) and surface areas (mean $=8.20 \times 10^{7} \mathrm{~nm}^{2}$ ) varied over a wide range and were strongly correlated, the latter consistent with structures built largely of narrow tubes (Fig. 8A). Holes (up to seven on a single spine) were found on $37 \%$ of spines and were more prevalent in larger spines (Fig. 8B). Some holes were formed by tight junctions between two membrane-bound filopodial extensions while others appeared as cytoplasmic continuities (Fig. 9). The number of active zones per spine ranged from 1 to 49 and was strongly correlated with size (Fig. $8 C$ ) and number of holes (Fig. $8 D$ ). These 
A

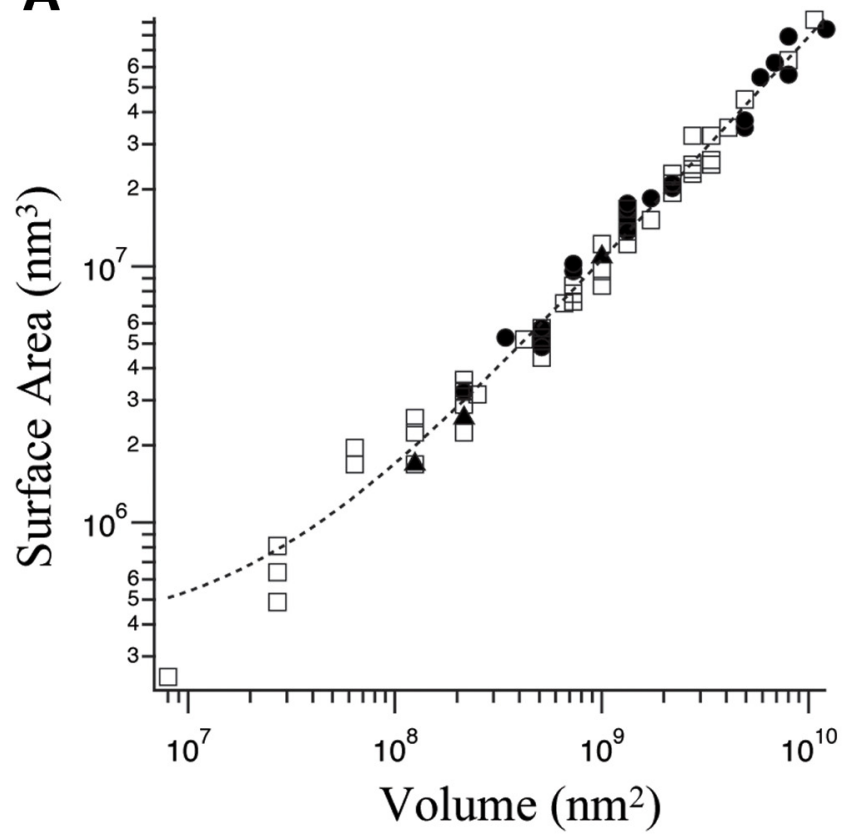

C

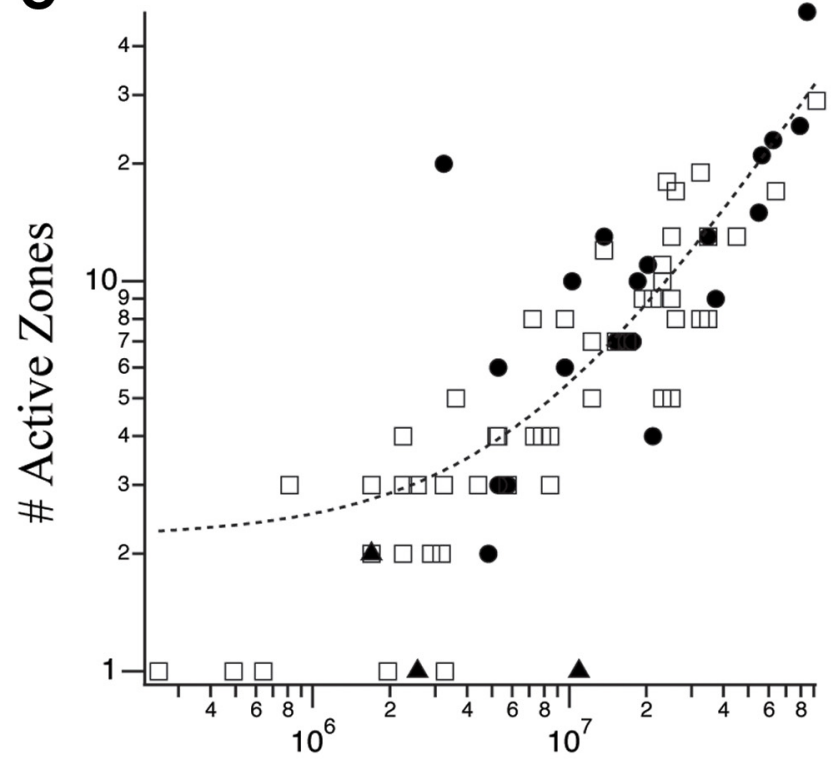

Surface Area $\left(\mathrm{nm}^{2}\right)$
B

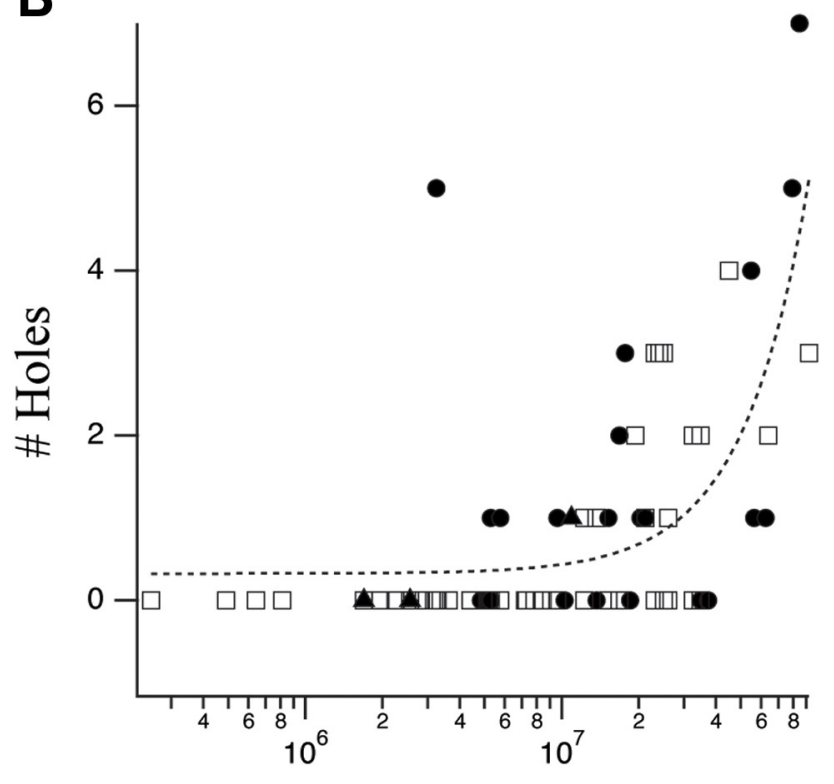

Surface Area $\left(\mathrm{nm}^{2}\right)$

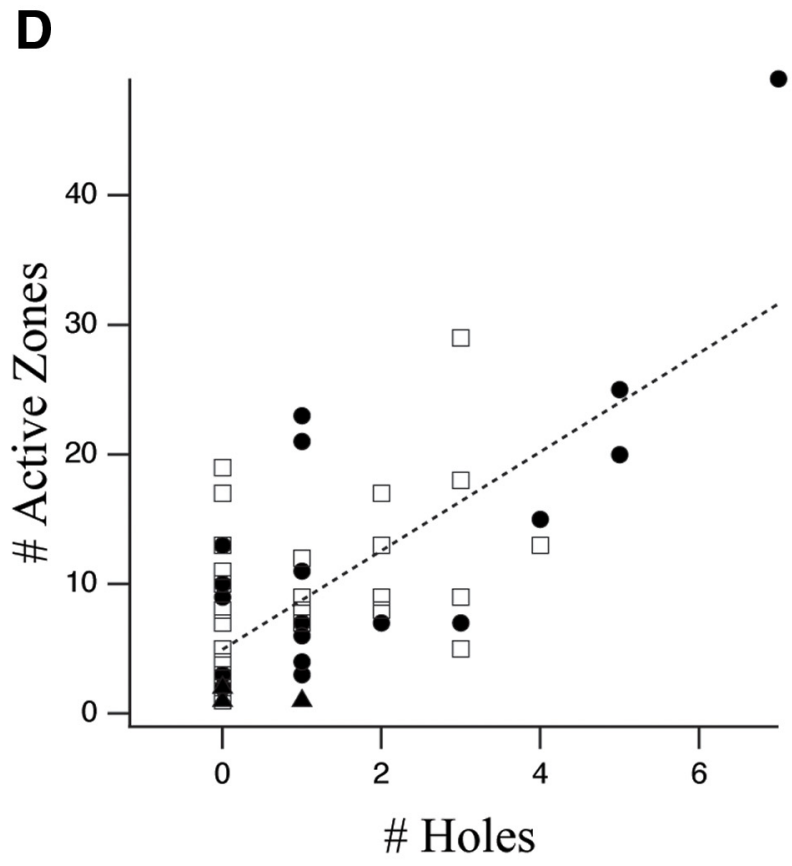

Figure 8. Morphometric analysis of the toric spine population. Volume, surface area, number of holes and number of active zones were quantified for each toric spine $(n=76)$ with 52 located on dendrites (open squares), 21 on the soma (filled circles), and 3 on the thin process (filled triangles). $\boldsymbol{A}$, Volume versus surface area. Data were marginally better fit (dashed line) by a power law $\left(\chi^{2}=8.15 \times 10^{14}\right)$ than linear regression $\left(\chi^{2}=9.28 \times 10^{14} ; r^{2}=0.97, p<0.00001\right.$. B. Holes versus surface area: marginally better fit (dashed line) by power law $\left(\chi^{2}=90.5\right)$ than linear regression $\left(\chi^{2} 91.1 ; r^{2}=0.42, p<0.00001\right)$. $\boldsymbol{C}$, Active zones versus surface area: marginally better fit (dashed line) by linear regression $\left(\chi^{2}=1254 ; r^{2}=0.73, p<0.00001\right)$ than power law $\left(\chi^{2}=1282\right)$. $D$, Active zones versus holes: best fit by linear regression $\left(r^{2}=0.50, p<0.00001\right)$. There was a weak tendency for spines located on the soma to be larger than those located on dendrites, in both volume (mean values 3.13 vs $1.62 \times 10^{9} \mathrm{~nm}^{3} ; p=0.026$ Mann-Whitney $U$ test) and surface area (mean values 2.75 vs $1.50 \times 10^{7} \mathrm{~nm}^{2} ; p=0.016$ Mann-Whitney $U$ test). Spines on the thin process tended to be smaller though there were not enough data points to make a statistical comparison.

data demonstrate large morphologic diversity within the toric spine population.

To determine patterns of synaptic convergence, 27 spines representing the morphologic spectrum were se- lected for reconstruction of axonal inputs. Three examples are shown in Figure 10. In each case, the active zones derived from multiple axons: 10 axons provided 25 AZs to TS1; three axons provided six AZ to TS2; and 10 


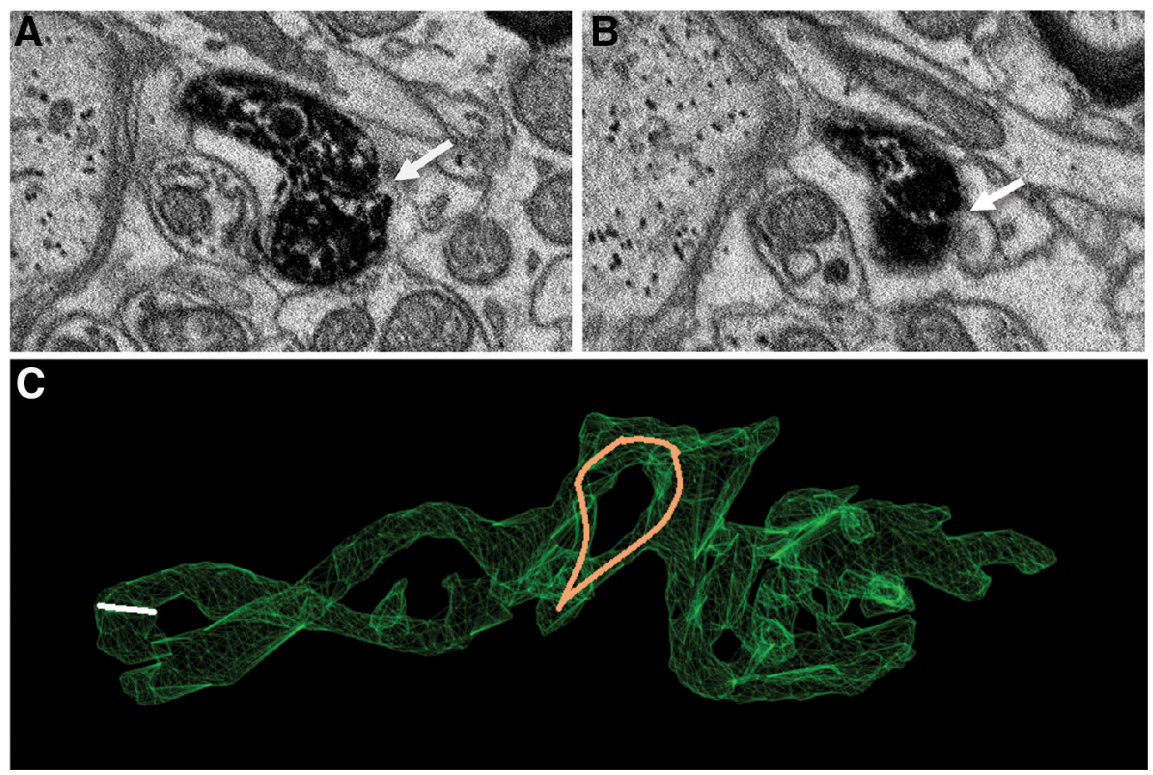

Figure 9. Examples of tight junction and cytoplasmic continuities. $\boldsymbol{A}, \boldsymbol{B}$, Consecutive EM sections showing a torus formed by a tight junction (white arrows). $\boldsymbol{C}$, Location of the tight junction shown above on TS1, indicated by white line. A separate torus exhibited cytoplasmic continuity across all sections (pale loop).

axons provided 23 AZ to TS7. In fact, the majority of toric spines (all except three of the 27 representative spines) received more than one axonal input. The number of axonal inputs strongly correlated with the number of AZs, and this was true for spines located on the soma or dendrites; spines on the soma tended to receive more inputs than those on the dendrites or thin process (Fig. $11 A$ ), consistent with their tendency to be larger.

To determine the degree to which inputs to a single spine were balanced or unbalanced in anatomical strength we measured the input fraction, defined as the number of active zones made by a single input divided by the total number of active zones received (metric range = $0-1$ ). A low fraction indicates the input has a weak contribution to the overall innervation of the spine, while a high fraction indicates the input is a dominant driver. The distribution of input fractions was skewed low (mean $=0.20$, $\mathrm{SD}=0.19, n=138$ ) indicating the most common arrangement was integration of many individually weak inputs, a situation of anatomical balance (Fig. 11B). Large input fractions were mostly limited to spines that received few inputs (Fig. 11C) and active zones (Fig. 11D), both of which set a high lower limit on

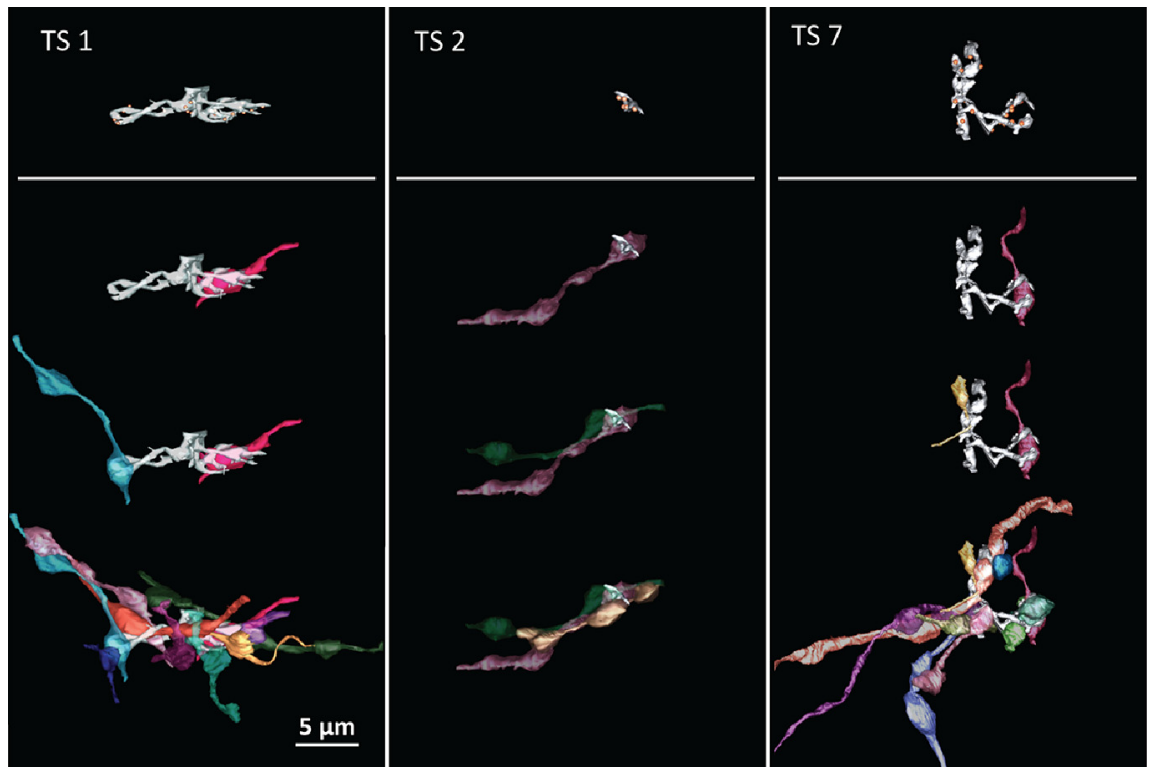

Figure 10. Toric spines receive multiple presynaptic inputs. Representative examples of axonal convergence onto three toric spines for TS1, TS2, and TS7. Top panels depict the spines (light gray) with all of its active zones (yellow circles). The lower panels show one, two and all axonal inputs (10,3, and 10, respectively) added sequentially in color. 
A

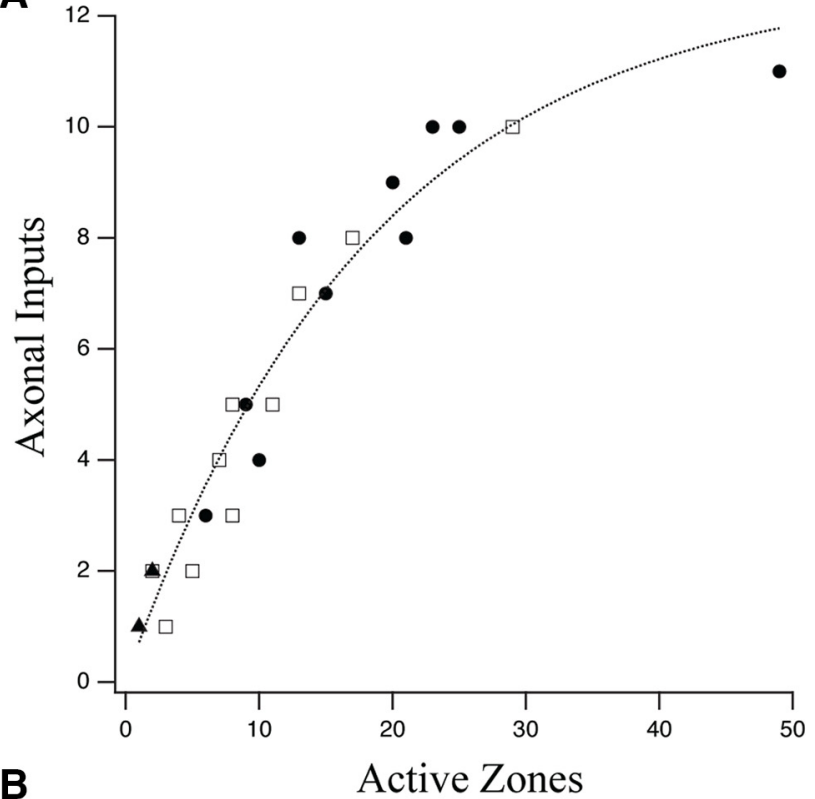

B

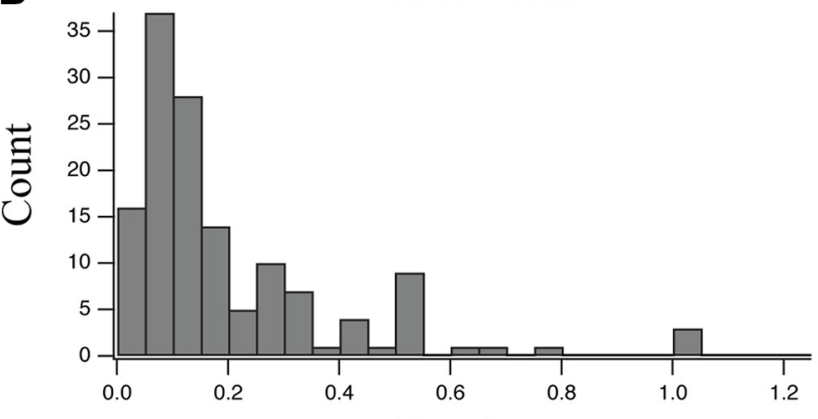

C

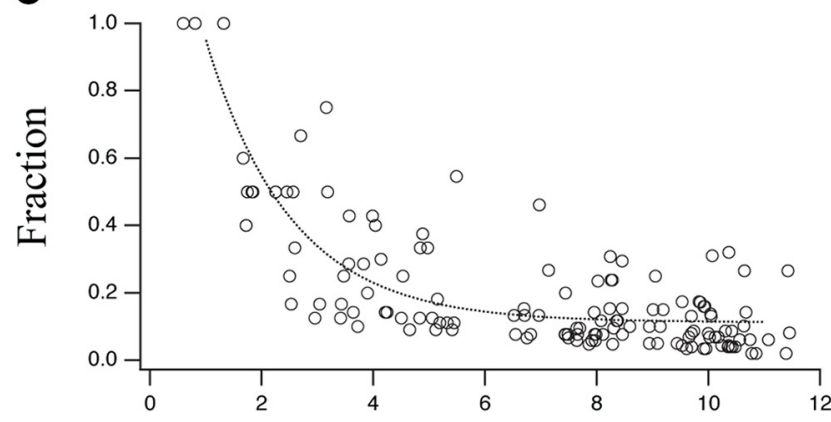

D

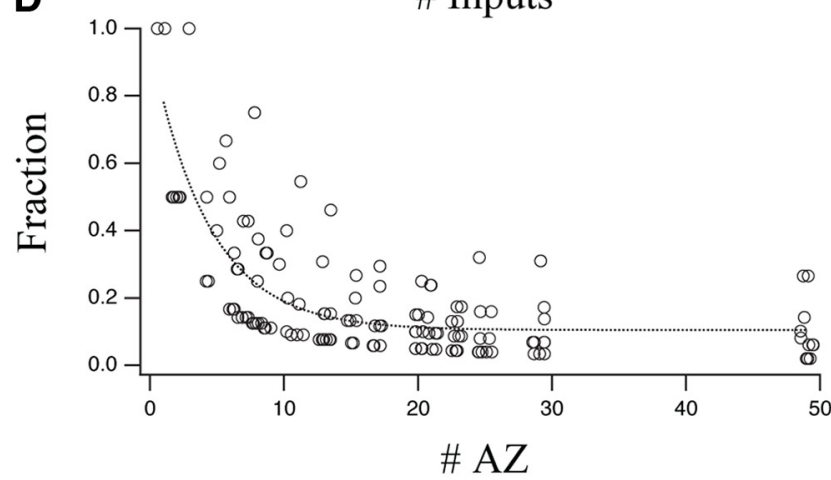

Figure 11. Synaptic convergence onto toric spines. Quantification of synaptic convergence for 27 representative toric spines:
Figure 11. continued

13 located on dendrites (open squares), 11 on the soma (filled circles), and 3 on the thin process (filled triangles). $\boldsymbol{A}$, The number of axonal inputs strongly correlated with the number of active zones (linear regression: $r^{2}=0.813, p \leq 0.00001, \chi^{2}=49.4$ ) and the data were better fit by an exponential function $\left(\chi^{2}=14.59\right.$; dotted line). This was also true for both subpopulations of toric spines on the dendrites (linear regression: $r^{2}=0.895, p \leq$ $0.00001, \chi^{2}=8.88$; exponential fit $\chi^{2}=6.177$ ) and on the soma $\left(r=0.837, r^{2}=0.700, p=0.00125, \chi^{2}=25.46\right.$; exponential fit $\left.\chi^{2}=6.65\right)$. There was a weak tendency for spines located on the soma to have more inputs (mean values 7.1 vs $4.3 ; p=0.03$ Mann-Whitney $U$ test) and active zones (17.9 vs $8.9 ; p=0.026$ Mann-Whitney $U$ test) than those on the dendrites. $\boldsymbol{B}$, Histogram of the fraction of total synaptic input to each spine (see Materials and Methods) contributed by each axonal input $(n=138)$. C, Input fraction decreased exponentially (dotted line) as the number of inputs increased. $\boldsymbol{D}$, Input fraction decreased exponentially (dotted line) as the number of active zones increased. For both $\boldsymbol{C}, \boldsymbol{D}$, the $x$-coordinate was randomly shuffled by \pm 0.5 for display purposes only.

the possible values of input fraction. Overall, the common motif was anatomically balanced integration.

To determine whether multiple inputs derived from the same parent axon, seven spines whose axons had been extended for considerable distance without ambiguity were selected for trajectory analysis (mean length $=20.6$ $\mu \mathrm{m}$; see Materials and Methods; Fig. 12A,B). None of these 40 axons joined together. Further, the population of trajectories was distributed near randomly in 3D (Fig. $12 C$ ), and this divergent profile (Figs. 12D, 13) was evident at individual spines (with one exception) including all of the most complex spines. The axonal segments tended to exhibit minimal curvature between incoming and outgoing ends (Fig. 14). In total, wide divergence of axons with linear trajectories strongly suggests that the axons originate from diverse input sources (see Discussion).

To investigate the potential for toric spines to form synapses with novel inputs we investigated the CF of two complex spines, TS1 and TS7. Prior work defined "filling fraction" as the number of synapses formed on a postsynaptic target (in that case, a dendrite or neuron) divided by the total number of synapses available within a local volume of surrounding neuropil extending $2 \mu \mathrm{m}$ in all directions away from the postsynaptic target (Stepanyants et al., 2002). This is the typical range of filopodial extensions that occur across circuits and species in healthy tissue undergoing experience-dependent synaptic plasticity (Grutzendler et al., 2002; Trachtenberg et al., 2002). We define a closely related concept, CF, which is the number of axons connected to the spine divided by the total number of axons that could be connected by virtue of proximity and lack of intervening myelin (Mishchenko et al., 2010). To determine CF, all unmyelinated axons within two $\mu \mathrm{m}$ of the target spine were reconstructed (Fig. 15A). Infiltrating TS1 were 61 unmyelinated axons, 10 of which were synaptically connected to it (CF $=0.164$ ). For TS7 the numbers were 64 axons, 10 connected, CF $=0.156$. These low CFs are on par with prior reports of filling fractions for circuits in mammalian 

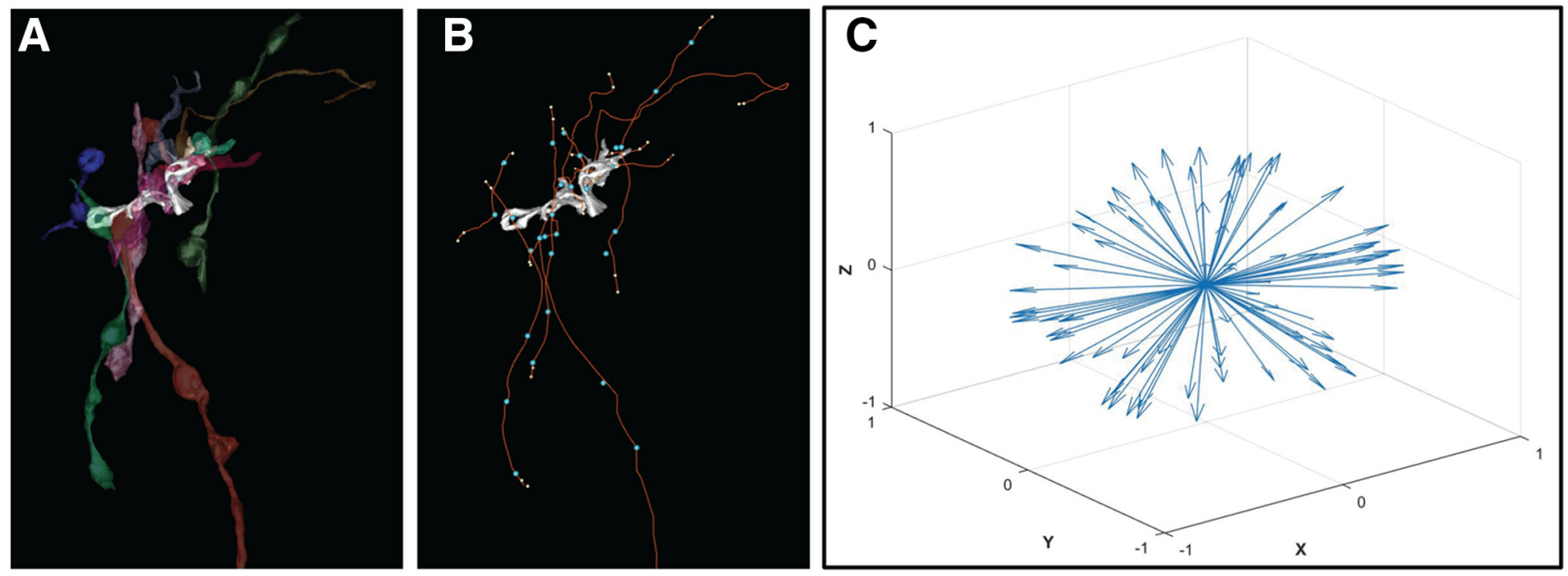

D

TS1

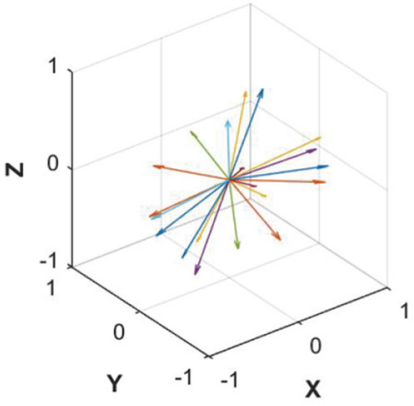

TS48

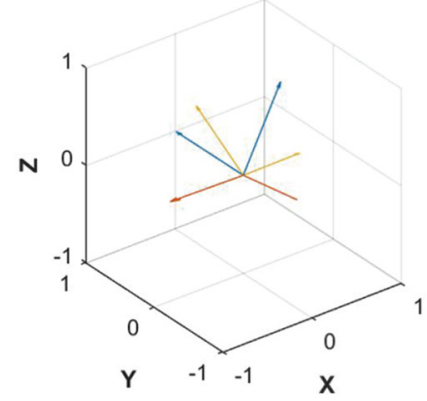

TS2

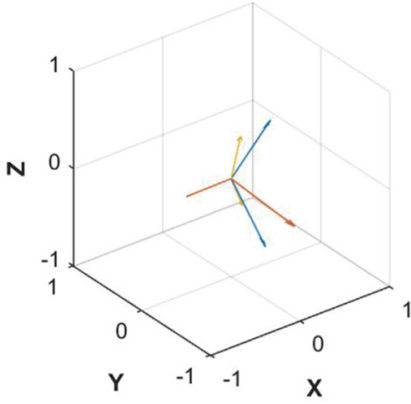

TS67

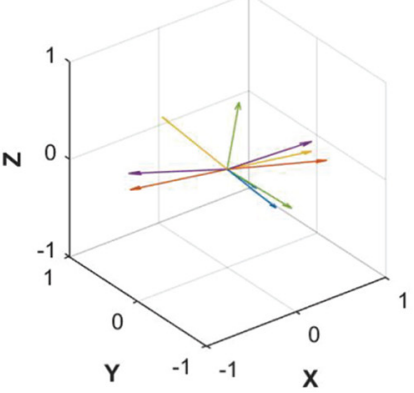

TS7
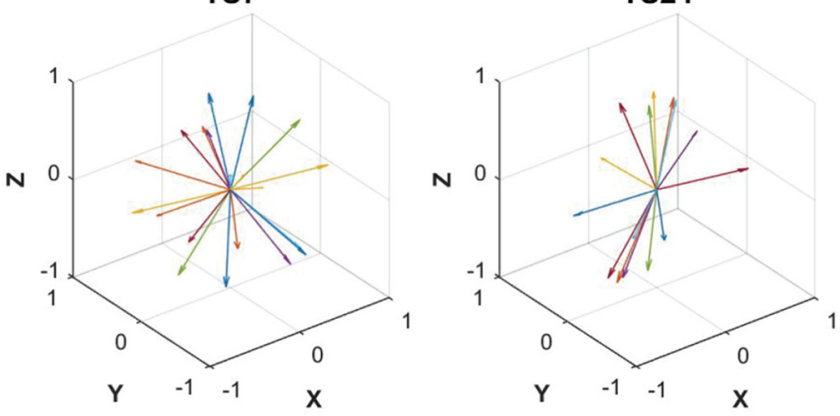

TS76

Figure 12. Trajectory analysis of axonal inputs. $\boldsymbol{A}$, Representative example trajectory analysis. Axons providing input (in this case to TS1) were extended to the edge of the volume or until a reconstruction ambiguity was encountered. $\boldsymbol{B}$, For a total of 40 axons synapsing onto seven toric spines, the length (red line), number of boutons (blue dots), and the outgoing trajectories at each end of the axon (small yellow dots) were measured (see Materials and Methods). $\boldsymbol{C}$, Axonal trajectories were represented as vectors through the two end points, scaled to a length of one and plotted in three dimensions with their tails at the origin $(n=81)$. $D$, In plots $1-7$, the vectors are grouped by spine and the ends of the same axon are paired visually by color within each plot.

tissue, e.g., 0.18 and 0.19 for two densely reconstructed hippocampal dendrites (Mishchenko et al., 2010).

Synaptic connectivity of the infiltrating axons could in principle be random or structured. To test this, we constructed a bootstrap analysis based on the assumption that each axon has equal ability to form an active zone with the target spine during a hypothesized epoch of microstructural plasticity. We determined the probability envelope for distributing the total number of $A Z s$ received (25 for TS1, 23 for TS7) across each potential axonal input
(61 for TS1, 64 for TS7). The actual data fell well outside the probability envelope based on random access (Fig. $15 C)$. This indicates that connectivity is structured such that certain axons have, or develop, preferential access. Such non-random connectivity is also found in mammalian cortex (Kasthuri et al., 2015) and thalamus (Morgan et al., 2016), suggesting a common principle for circuit development.

To open a path for linking microanatomical patterns of synaptic convergence with functional measurement of neu- 

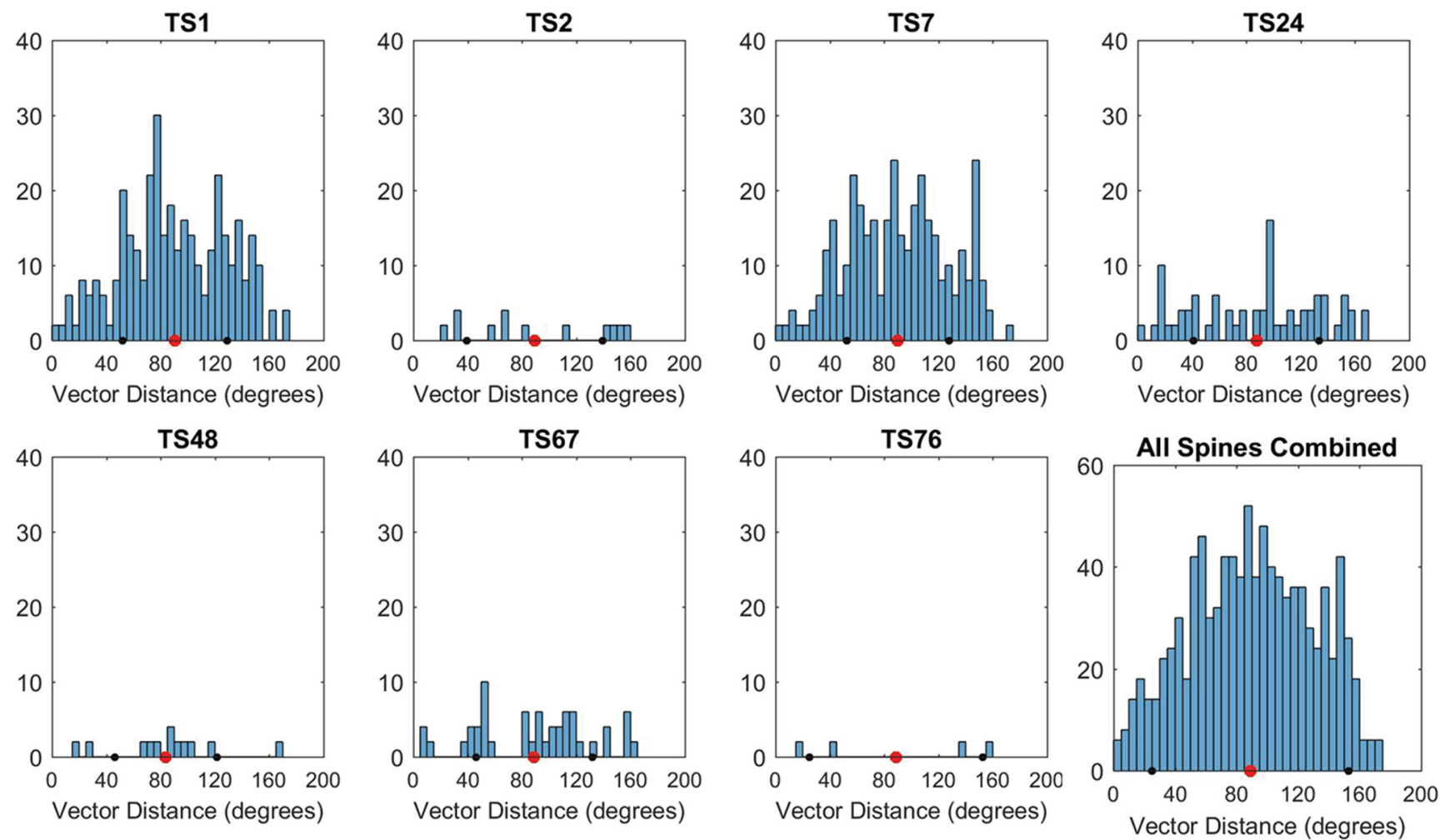

Figure 13. Angular distances between the input trajectories onto toric spines. Histograms of the angular distance between each trajectory vector and every other vector for each spine, excluding the vector from the other end of same axon. Distances were calculated with the dot product (see Materials and Methods). Mean across all spines $=89.7^{\circ} ; \mathrm{SD}=40 ; N=78$ vectors, 976 pairings; bin width $=5$ ). A population of random trajectories predict a Gaussian distribution of distances with a mean of $90^{\circ}$, as observed from $6 / 7$ spines and the overall population, while a population of bundled trajectories predicts a bimodal distribution with a mean of $90^{\circ}$, as observed for TS76.

ronal computation, we developed an ex vivo slice preparation to record from space-specific neurons. Recording electrodes were targeted to large somata in ICX under visual guidance (Fig. 16A) and used to record membrane potential responses using a current-clamp protocol (Fig. $16 B$ ). Neurons ( $n=20$ from five owlets) exhibited a spec-

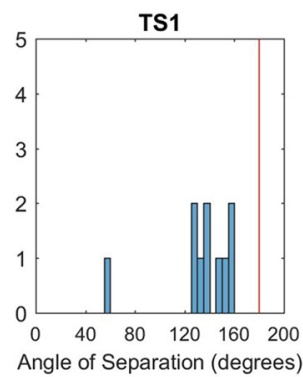

TS48

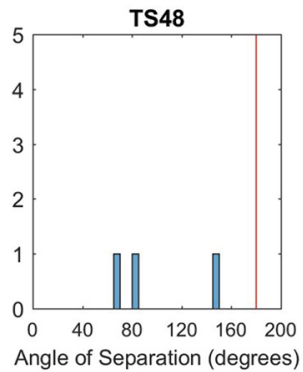

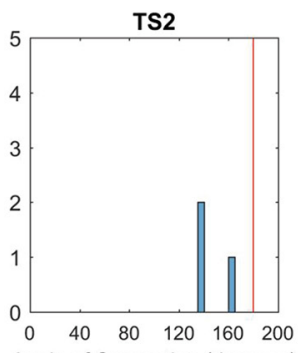

TS67

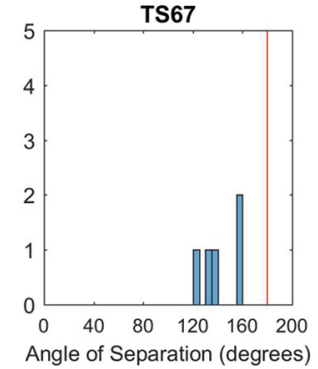

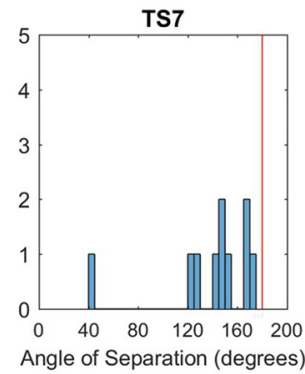

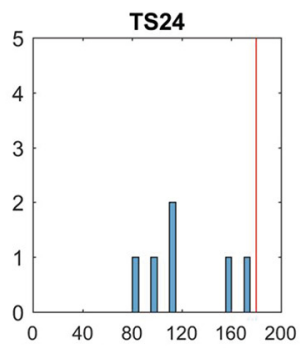

TS76

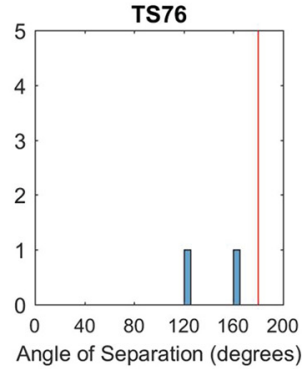

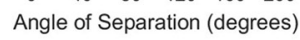

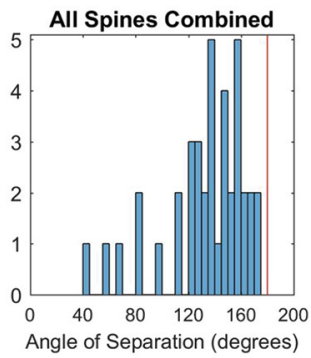

Figure 14. Angle of separation between paired ends. Histograms of the angular distance between the paired ends of each axon. Mean $=132.9^{\circ} ; \mathrm{SD}=31.5 ; N=40$. Linear trajectories predict a clustered distribution near $180^{\circ}$, as observed for the overall population, while highly curved trajectories predict a Gaussian distribution with a mean near $90^{\circ}$. 

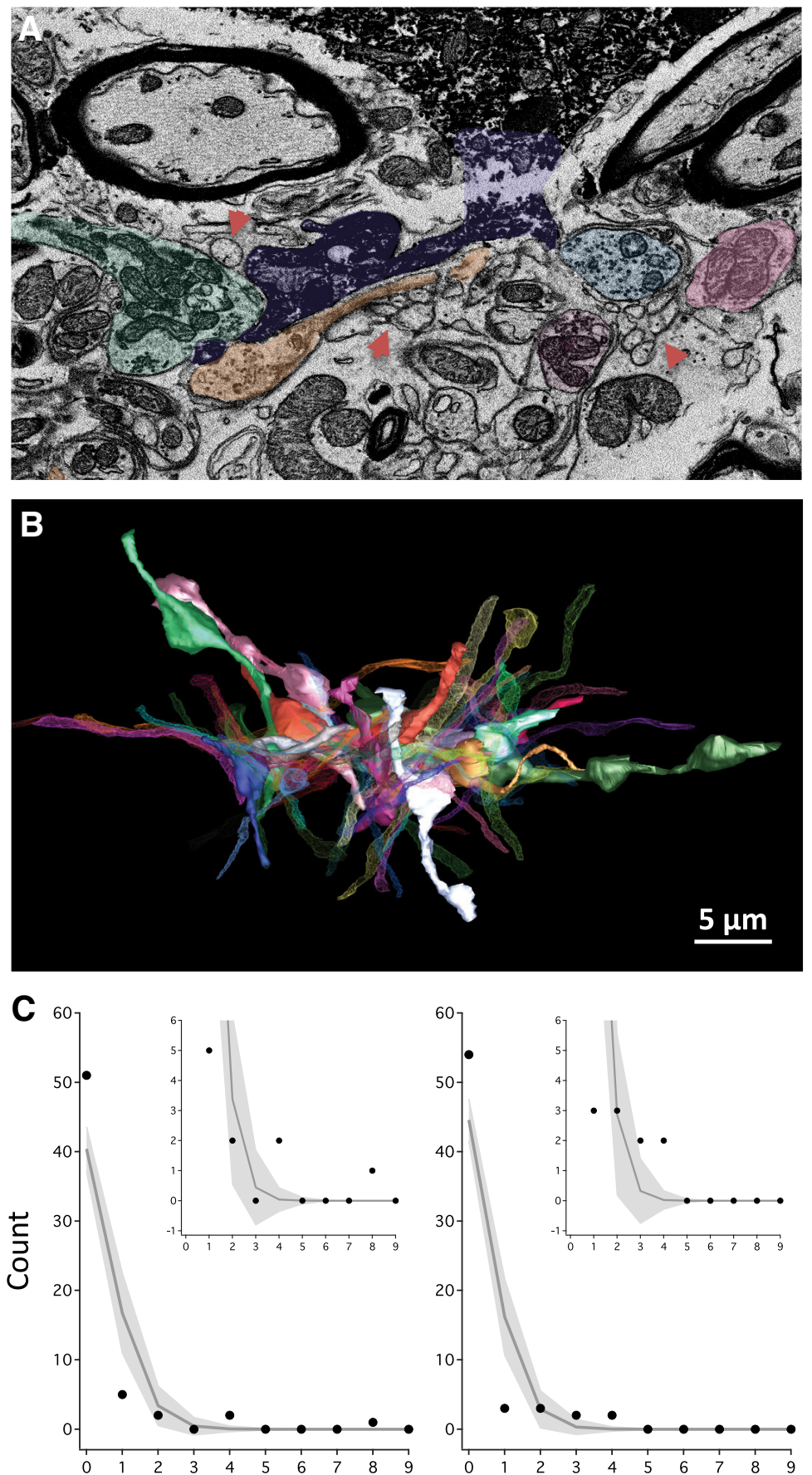

\section{Active zones per axon}

Figure 15. Potential connectivity of toric spines. To assess potential connectivity of TS1 and TS7, all unmyelinated axons that passed within $2 \mu \mathrm{m}$ of the spine were reconstructed. A, SBEM section showing four annotated axons (shaded in color) synapsing onto TS1 (dark purple). Arrows point to nearby axons which do not make any synapses onto TS1. B, All 61 unmyelinated axons within $2 \mu \mathrm{m}$ of TS1 (solid gray). There were also five myelinated long-range axons that came within $2 \mu \mathrm{m}$ of TS1 (data not shown and excluded from this analysis; see text for explanation). In all, 10 unmyelinated axons synapsed onto TS1 (solid axons) and 51 did not (meshed axons). Thus, the CF for TS1 is 0.164 . The CF of TS7 (data not shown) is 0.156 (10 connected axons, 54 unconnected axons). $\boldsymbol{C}$, Bootstrap analysis of potential connectivity of TS1 (left panel) and TS7 (right panel). Histograms indicate the relative frequency of observing axons connected with the specified number of active zones to TS1 in the real data (solid circles) and 10,000 simulations (gray line; light gray envelope indicates $\pm 2 \mathrm{SD}$ ). In both spines, four of the 10 data points lie outside the envelope of expectation based on random connectivity. Insets display the same data over a magnified range. 
A

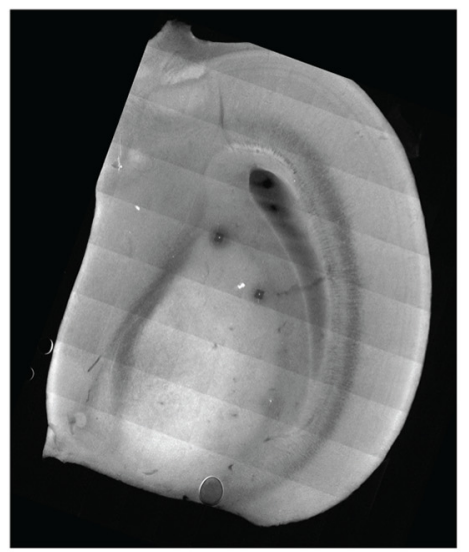

C

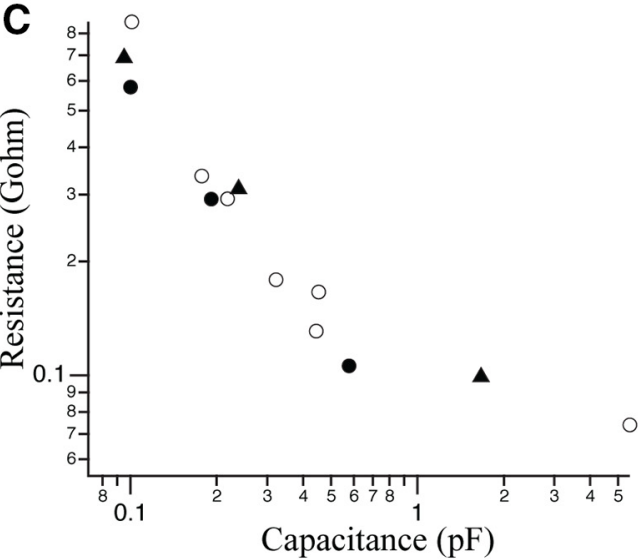

E

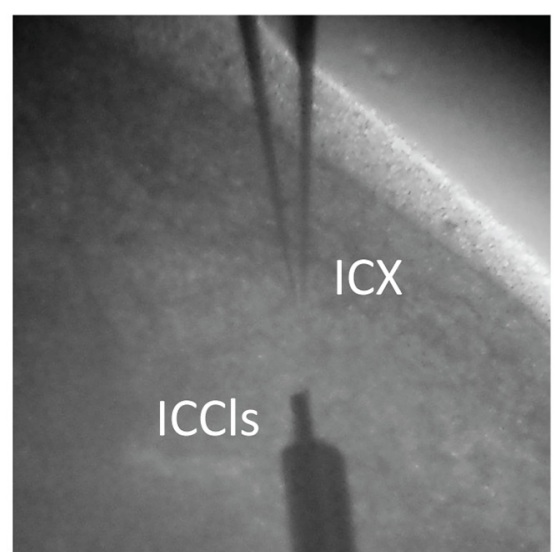

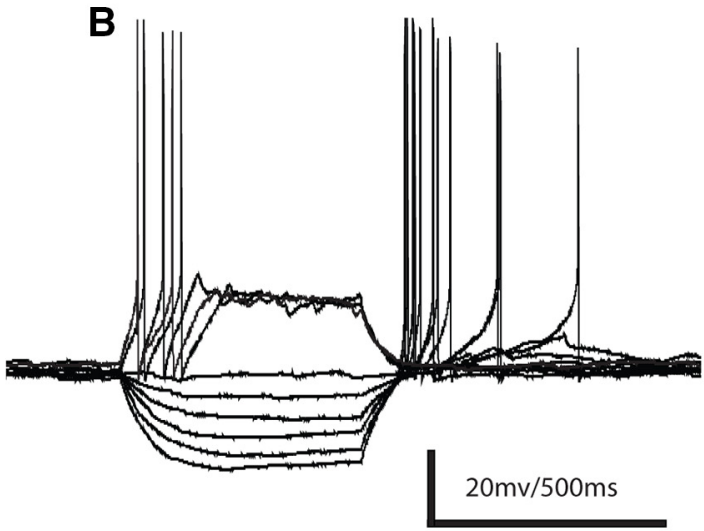

D

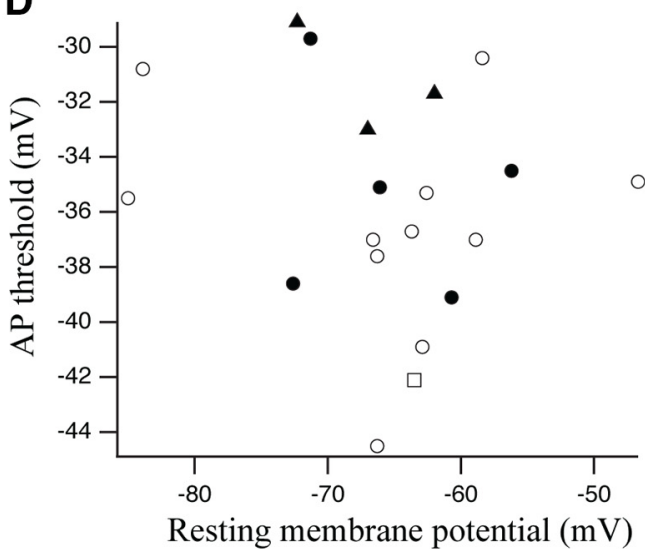

$\mathbf{F}$

- Control

- AMPA receptor antagonist

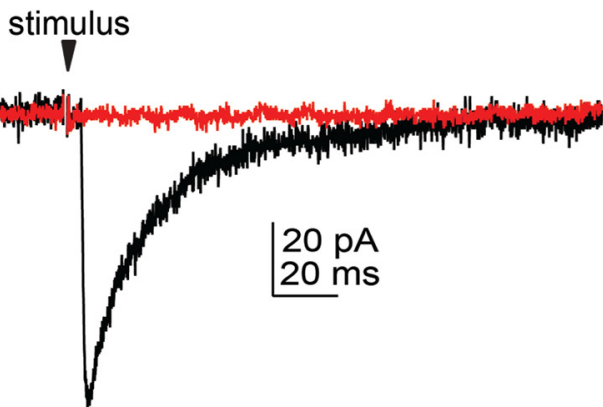

Figure 16. Patch clamp recordings. A, Mosaic image of a $300-\mu \mathrm{m}$-thick acute horizontal section through the tectal lobe. Photobleached squares reveal locations of recorded neurons in ICX. $\boldsymbol{B}$, Example of current step protocol used to measure intrinsic membrane properties ( $n=20$ cells, five slices, six owlets age 35-40 d). $\boldsymbol{C}$, Resistance versus capacitance for 13 cells. $\boldsymbol{D}$, Action potential (AP) threshold versus resting membrane potential for 20 cells. For both $\boldsymbol{C}$ and $\boldsymbol{D}$, filled circles $=$ cells with toric, atypical, and typical spines $(n=5)$; filled triangles = cells with atypical and typical spines but not toric spines $(n=3)$; open squares $=$ cells with no spines $(n=1)$; open circles = not determined $(n=11)$. $\boldsymbol{E}$. Electrical stimulation experiment with glass recording electrode in ICX and bipolar stimulating electrode in ICCls. $\boldsymbol{F}$, Example of electrically-evoked EPSC $(n=2)$ that was completely abolished by bath application of AMPA receptor antagonist NBQX.

trum of intrinsic membrane properties (Fig. 16C,D; Table 2). Some neurons $(n=16)$ were filled with biocytin for retrospective imaging to determine cell type. In this population of neurons in juvenile owls, large atypical spines were prevalent including toric-like structures of narrow tubular build with multiple arms whose ends often infolded on themselves, but which infrequently presented as closed holes (Fig. 17D). The morphologic diversity was greater than in the adult space-specific neuron population, with many juvenile cells expressing some mixture of 
Table 2. Patch-clamp recordings

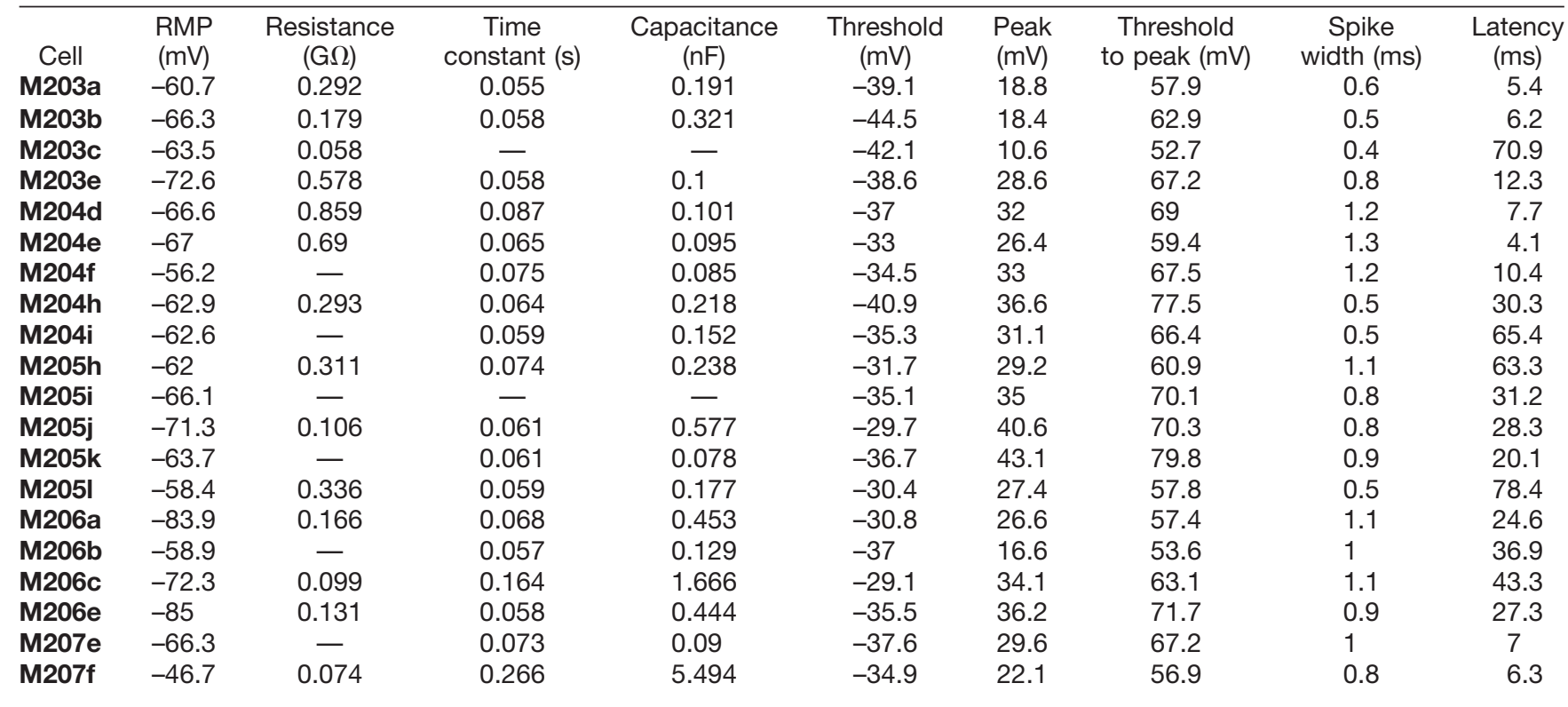

Intrinsic membrane properties recorded from 20 cells across five juvenile owls age $35-40$ d. All reported data are from cells that passed multiple quality controls.

atypical and typical spines (Fig. 17A,B), while a minority ( $n$ $=5$ ) lacked a high density of spines (Fig. 14C). Therefore, juvenile cells were not classified as Type I or Type II, but instead scored for the presence $(n=7)$ or absence $(n=9)$ of toric-like spines. There was a trend for juvenile cells with toric-like spines to have larger soma and thicker primary dendrites than those lacking them (Fig. 17E-G), although these differences were not statistically significant, and both juvenile and adult cells had on average the same number of primary dendrites (mean $=4.5$ for both), a comparative profile similar to the differences between Type I and Type II adult cells (Fig. 2). In total, the juvenile cells appeared as developmental precursors to toric space-specific neurons. Finally, in separate voltage-clamp experiments, electrical stimulation of the known input source to ICX, the ICCls, evoked typical AMPA-mediated EPSCs (Fig. 16E,F; $n=2$ ), consistent with all prior studies of this monosynaptic connection.

\section{Discussion}

We describe the morphology and synaptic innervation patterns of a novel brain structure, toric spines. Across circuits and species, toric spine morphology appears unique. In contrast, while their high integrative capacity is unparalleled in degree, it is evocative of reports of complex spine types found in mammalian thalamus, hippocampus and cerebellum. This suggests that toric spines may represent a standout example of a processing motif common across the animal kingdom. The discussion below critically analyzes toric spine morphology, integrative capacity, implications for cellular computation, potential role in plasticity, and concludes by outlining testable hypotheses to probe more deeply into their structure and function.

Both STED and SBEM imaging revealed a broad spectrum of spine morphologies. Features that varied mark- edly across the population include size, number of holes and degree of branching. Features that tend to be shared include narrow tubular build, paucity of bulbous heads, and wide necks at the process interface. This profile distinguishes toric spines from all previously documented spine types including typical thin, stubby, mushroom (Bourne and Harris, 2007; Chirillo et al., 2019) or branched spines (Bourne and Harris, 2008), and atypical dendritic structures including thorny excrescences, dendritic appendages or glomerular excrescences (Jones and Powell, 1969; Spacek and Lieberman, 1974; Rapisardi and Miles, 1984; Erişir et al., 1997; Morgan et al., 2016). Perhaps most striking, toric spines received up to 49 active zones contributed by up to 11 axonal inputs - a stark contrast to typical spines (e.g. Arellano et al., 2007) that compartmentalize single inputs or thorny excrescences which detonate their postsynaptic target (Table 3). Given their size and integrative capacity (discussed below), toric spines could be classified as atypical dendrites. We call them spines because, like typical spines, they are substantially smaller in caliber than the parent process, a size differential that exceeds the difference between higher and lower order dendritic branches.

The narrow tubular build of toric spines should produce a short length constant due to high axial resistance, and therefore predicts that single synaptic inputs made onto one spine are isolated from those onto another (Rall, 1974). At the same time neighboring inputs onto the same spine should be subject to summation. Compartmental simulations of the reconstructed space-specific neuron to test this intuition have so far been unsuccessful due computational limitations of available modeling platforms. In comparison, simulations of neocortical pyramidal cells reconstructed from light microscopy (i.e., lacking ultrastructural detail and therefore amenable to compartmen- 

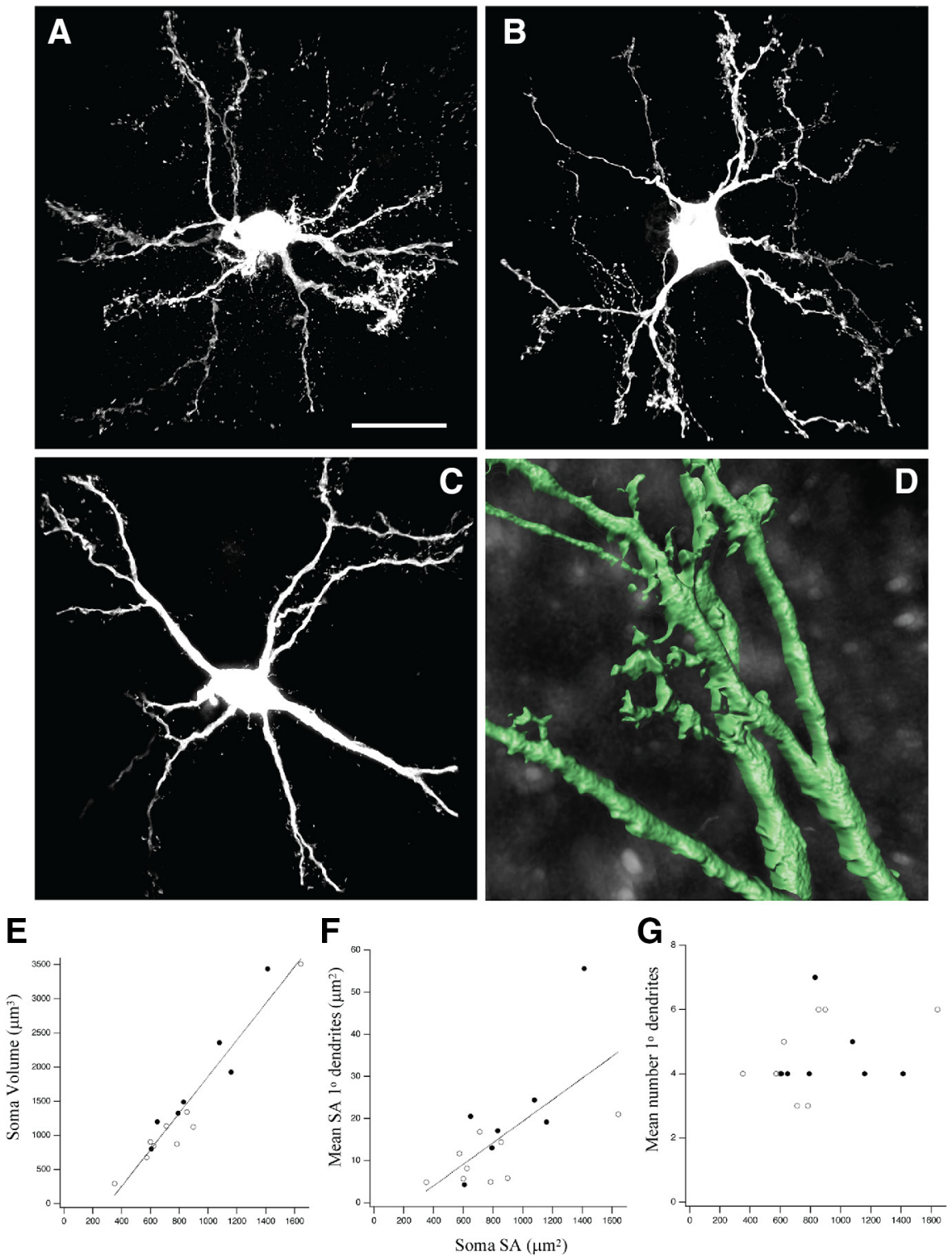

Figure 17. Morphologies of putative SSNs in juvenile owls. $\boldsymbol{A}-\boldsymbol{C}$, Examples of the morphologic diversity of ICX cells in juvenile owls. Cells studded with a variety of spine types, atypical, branched, toric-like, and typical, were common and resembled Type I adult SSNs in multipolar structure $(\boldsymbol{A}, \boldsymbol{B})$. A minority of juvenile cells exhibited relatively few spines $(\boldsymbol{C})$. Toric-like structures with incompletely closed holes $(\boldsymbol{D})$ were found on $7 / 16$ juvenile cells (filled circles in $\boldsymbol{E}-\boldsymbol{G}$ ). Scale bar $=30 \mu \mathrm{m}(\boldsymbol{A}-\boldsymbol{C})$ and $5 \mu \mathrm{m}(\boldsymbol{D})$. $\boldsymbol{E}$, Soma volume versus surface area across both types: $r=0.962, r^{2}=0.925, p<0.00001$. $\boldsymbol{F}$, Mean cross sectional surface area of primary dendrites versus surface area of the soma: $r=0.676, r^{2}=0.457, p=0.004$. G, Mean number of primary dendrites versus surface area of the soma: $r=0.323, r^{2}=0.104, p=0.222$.

tal simulation) have shown that neuronal computation can be represented by a two-stage model in which individual dendritic branches perform the first-stage processing of summation of EPSPs (Poirazi et al., 2003). Active properties (voltage-gated ion channels) modify the rules of dendritic integration providing a range of operations from supra-linear to sub-linear. The second stage is linear summation of dendritic branch output at the soma. The multipolar structure of space-specific neurons is well suited to emulate this processing scheme with the significant addition of an earlier-stage operation at toric spines.
Direct tests of these computational strategies require whole-cell recording in combination with targeted synaptic stimulation. We demonstrate, for the first time to our knowledge, the ability to make high-quality patch-clamp recordings from ICX neurons. Development of this ex vivo preparation opens a path for understanding the casual connections between microanatomical convergence and cellular computation.

All large toric spines with analyzed convergence patterns $(n=24)$ were found to receive synaptic input from multiple axons, indicating that they are structured to act as integrators. Synaptic convergence onto branched spines 
Table 3. Comparison of spine types

\begin{tabular}{llll}
\hline & Typical mushroom & Thorny Excrescence & Toric \\
Size & Small & Large & Large \\
Neck & Thin & Thin & Wide \\
Spine head & Large & Large & Rarely apparent \\
Branched? & Rarely & Profusely & Sometimes \\
Holes? & No & No & Yes \\
Cellular location & Dendrites & Base of apical dendrite & Soma and dendrites \\
Synapses per spine & $\sim 1$ & $10+$ & $10+$ \\
Axonal inputs & $\sim 1$ & $1-2$ & Up to 11 \\
Function & Compartmentalization & Detonation & Integration
\end{tabular}

The morphologies, dimensions, patterns of synaptic convergence, and known or hypothesized functions are shown for typical mushroom spines (e.g. Arellano et al., 2007, their Fig. 2) thorny excrescences (e.g. Wilke et al., 2013, their Fig. 5) and toric spines.

and other atypical dendritic protrusions has been suggested, though not firmly established, by earlier studies. Pioneering work using serial EM to reconstruct rat cerebellar Purkinje cells demonstrated a complex spine type with up to five branches. Independent axonal boutons could be found on distal branches of the same spine, several microns apart, raising the possibility that branched spines receive inputs from multiple axons, though this remains untested (Harris and Stevens, 1988). The prevalence of branched spines on Purkinje cells has been estimated between $2 \%$ and $~ 6 \%$ (4/64; Harris and Stevens, 1988; Lee et al., 2004) and $\sim 15 \%$ on adult-generated granule cells in the dentate gyrus, where they are also polyinnervated (Bosch et al., 2015), suggesting an important role in neuronal computation. Synaptic glomeruli in mammalian thalamus receive convergent input from anatomically and neurochemically diverse sources (Jones and Powell, 1969; Spacek and Lieberman, 1974; Erişir et al., 1997). In a recent connectomics study, two densely reconstructed glomeruli received input from 15 different thalamocortical afferents (Morgan et al., 2016), a level of convergence similar to that observed in our dataset. The central postsynaptic compartment of glomeruli are dendritic shafts from which multiple tiny excrescences emerge, distinct electrotonically from toric spines. Nonetheless, the above results in combination with our findings raise the intriguing possibility that synaptic convergence onto complex spine types is more common than currently appreciated.

The conclusion that toric spines are innervated by multiple input sources is supported by analysis of axonal convergence onto 27 spines and trajectory analysis for seven. Not one shared branchpoint was found (40 axons, mean length $=20.6 \mu \mathrm{m})$. Until the axons are traced back to their parent cell bodies, a single source explanation cannot be logically eliminated. We think it is highly unlikely because it would require parent axons to branch, diverge at least $10-100$ s of micrometer, then re-converge and terminate within an extremely small volume of tissue. There is no evidence for such an arrangement. Owls are known for unusual axonal specializations, delay lines (Carr and Konishi, 1988), yet these are found in the projection from nucleus magnocellularis to nucleus laminaris (Carr and Konishi, 1990), distant brainstem structures, not ICX. In fact, ICCls axons, which provide the bulk of input to ICX, exhibit typical terminal fields in which axonal branches diverge, not converge (DeBello et al., 2001; Rodriguez-
Contreras et al., 2005; McBride et al., 2008; McBride and DeBello, 2015). In principle, short branchlets with terminal boutons could be within range of a toric spine, but because those branchpoints are mere microns away from the parent axon, such arbors could not have contributed to the divergent trajectories of much longer axonal segments observed in our dataset.

The fact that individual ICCls axons cannot account for the convergence results does not rule out ICCls axons as a potential input source to toric spines. In fact, spacespecific neurons are known to receive input from multiple ICCls neurons encoding different sound frequencies. Integration across frequency is required to eliminate phase ambiguity and ultimately provide the owl with an accurate estimation of sound source azimuth. At the level of spiking output, frequency convergence is a non-linear operation. Thus, one hypothesis is that axons originating from multiple ICCls neurons encoding the same ITD but different frequencies converge onto individual toric spines where their EPSPs are combined and thresholded (Peña and Konishi, 2000, 2002). These predictions are testable using dual anterograde injections placed at different dorsoventral laminae, and whole-cell recordings, respectively.

A second possibility is that toric spines integrate ITD and ILD, a multiplicative operation. Binaural integration first occurs in the ICCls and is largely inherited by spacespecific neurons in ICX. Yet the precise anatomical and functional boundaries between ICCls and ICX are unclear. Several cytoarchitectonic markers delineate the lateral border of ICX, and separately, the border of the core of the central nucleus. But on close inspection of commonly used markers calbindin, calretinin and CaMKII, the ICClsICX border appears as a gradient. Microelectrode surveys also support the idea of functional heterogeneity along this border. Moreover, while cytoarchitectonic markers were not routinely employed in our study (see Materials and Methods), estimates based on comparison to similar sections from other owls indicate that at least some Type I space-specific neurons, including the one in the SBEM volume, were located in ICCls. This is a minor revision to the notion that IC-OT projection neurons are strictly confined to ICX. More importantly, it motivates the second hypothesis, that toric spines in ICCls receive convergent input encoding behaviorally relevant ITD-ILD pairs, from contralateral ICC (for ITD) and contralateral dorsal lateral lemniscal nucleus pars posterior (for ILD), and perform a 
multiplicative operation (Peña and Konishi, 2001, 2004) via a different complement of ion channels. A third possibility is that both hypotheses are true: toric spines are multipurpose integrators whose computation can be customized to cellular need.

Other potential sources of input to toric spines include the arcopallial gaze field (Cohen et al., 1998), which provides top-down attentional modulation and the intermediate/dOT, which provides a visually-based instructive signal to guide calibration of the auditory map (Hyde and Knudsen, 2000; Luksch et al., 2000). Both are anatomically sparse and unlikely to be dominant contributors. space-specific neurons do receive lateral connections from GABAergic neurons within ICX. The inputs to toric spines we observed, however, more closely resemble asymmetric glutamatergic synapses.

To our knowledge, this is the first report of a toric postsynaptic structure. Presynaptic perforations described extensively in the literature are cytoplasmically contiguous (Sorra et al., 1998). In contrast, at least some tori in our SBEM volume were interrupted by plasma membranes that appeared as sites of contact between two "arms" of the same spine (Fig. 9A,B). The simplest interpretation is these represent enduring adhesions between filopodial extensions emanating from the same base structure. These could be maintained or later converted to cytoplasmic continuities via anastomosis. Regardless of the developmental mechanics, the prevalence and functional properties of contact sites versus cytoplasmic continuities is expected to significantly impact current flow during synaptic stimulation.

The morphologies of ICX neurons from 35- to 40-d old juveniles labeled ex vivo and adult space-specific neurons labeled in vivo were qualitatively similar with a few clear differences: (1) juvenile cells often expressed a mix of atypical (branched and toric-like) and typical spines whereas adult cells were dominated by either toric (Type I) or typical (Type II) spines, but never both; (2) juvenile cells appeared smaller; and (3) juvenile cells sometimes appeared ciliated. We consider three interpretations: ex vivo artifact, unrelated cell type or developmental age.

Preparation of acute brain slices can induce changes in dendritic morphology in comparison to the in vivo condition as reflected in perfusion-fixed tissue (Fiala et al., 2003). In this scenario, toric-like spines would represent the sliceinduced disassembly of fully-formed toric spines. We think this is highly unlikely due to the number of neuropil elements involved in such a disassembly, specifically the high density of intertwining axons. Whole cell recordings in juvenile slices were targeted under visual guidance to the largest somatic profiles in ICX, the exact location of retrogradely labeled toric space-specific neurons in adults. That the measured size of juvenile cells was smaller is expected from the differential impact of immersion versus perfusion fixation. Specifically, measurements of three neuron types in primate hippocampus indicate that apparent soma volume is 1.36-2.7 times larger in perfusion-fixed than immersion-fixed tissue (Lavenex et al., 2009). On average, our adult cells (perfused brain) were 2.42 (volume) and 1.81 (surface area) times as large as our juvenile cells (immersed slices). Further, the one morphologic attribute not subject to differential shrinkage, number of primary dendrites, was the same in juveniles (4.5) and adults (4.7). In summary, the most straightforward interpretation is that the observed juvenile cell types represent developmental precursors of adult cell types.

Lesion reconstruction (Brainard and Knudsen, 1993), anatomic (Feldman and Knudsen, 1997; DeBello et al., 2001), and pharmacological (Feldman et al., 1996; Zheng and Knudsen, 1999) experiments pinpoint space-specific neurons as the cellular locus of learning (Knudsen, 2002). This raises the question of whether toric spines have a role in experience-dependent synaptic plasticity (DeBello et al., 2014). We found that toric spines are conspicuously well structured to be hubs for microstructural change: of all unmyelinated axons passing within $2 \mu \mathrm{m}$ of TS1 and TS7, only $\sim 16 \%$ were synaptically connected. Consequently, the number of different wiring diagrams that could be achieved by biologically plausible, small-scale dendritic extensions is very large (Stepanyants et al., 2002; Stepanyants and Chklovskii, 2005). We propose a model in which toric spines actively search for axonal inputs in their local environment, make "tester" synapses, then stabilize or eliminate these on the basis of efficacy in firing the postsynaptic cell (DeBello, 2008). This would provide a mechanism for pattern detection within a complex array of input sources, and the means to adapt connectivity to changing experiential needs. The high degree of cytoskeletal dynamics implicit in this model could also explain the broad spectrum of observed morphologies, with each spine stabilized not by genetic programs but by chance encounters of its extensions within a local, yet information diverse, axonal milieu.

\section{References}

Amaral DG, Dent JA (1981) Development of the mossy fibers of the dentate gyrus: I. A light and electron microscopic study of the mossy fibers and their expansions. J Comp Neurol 195:51-86.

Arellano JI, Benavides-Piccione R, Defelipe J, Yuste R (2007) Ultrastructure of dendritic spines: correlation between synaptic and spine morphologies. Front Neurosci 1:131-143.

Bergan JF, Knudsen El (2009) Visual modulation of auditory responses in the owl inferior colliculus. J Neurophysiol 101:2924-2933.

Bosch C, Martínez A, Masachs N, Teixeira CM, Fernaud I, Ulloa F, Pérez-Martínez E, Lois C, Comella JX, DeFelipe J, Merchán-Pérez A, Soriano E (2015) FIB/SEM technology and high-throughput 3D reconstruction of dendritic spines and synapses in GFP-labeled adult-generated neurons. Front Neuroanat 9:60.

Bourne J, Harris KM (2007) Do thin spines learn to be mushroom spines that remember? Curr Opin Neurobiol 17:381-386.

Bourne JN, Harris KM (2008) Balancing structure and function at hippocampal dendritic spines. Annu Rev Neurosci 31:47-67.

Brainard MS, Knudsen El (1993) Experience-dependent plasticity in the inferior colliculus: a site for visual calibration of the neural representation of auditory space in the barn owl. J Neurosci 13:4589-4608.

Brainard MS, Knudsen El, Esterly SD (1992) Neural derivation of sound source location: resolution of spatial ambiguities in binaural cues. J Acoust Soc Am 91:1015-1027.

Carr CE, Konishi M (1988) Axonal delay lines for time measurement in the owl's brainstem. Proc Natl Acad Sci USA 85:8311-8315.

Carr CE, Konishi M (1990) A circuit for detection of interaural time differences in the brain stem of the barn owl. J Neurosci 10:32273246. 
Chicurel ME, Harris KM (1992) Three-dimensional analysis of the structure and composition of CA3 branched dendritic spines and their synaptic relationships with mossy fiber boutons in the rat hippocampus. J Comp Neurol 325:169-182.

Chirillo MA, Waters MS, Lindsey LF, Bourne JN, Harris KM (2019) Local resources of polyribosomes and SER promote synapse enlargement and spine clustering after long-term potentiation in adult rat hippocampus. Sci Rep 9:3861.

Cohen YE, Miller GL, Knudsen El (1998) Forebrain pathway for auditory space processing in the barn owl. J Neurophysiol 79:891902.

DeBello WM (2008) Micro-rewiring as a substrate for learning. Trends Neurosci 31:577-584.

DeBello WM, Feldman DE, Knudsen El (2001) Adaptive axonal remodeling in the midbrain auditory space map. J Neurosci 21: 3161-3174.

DeBello WM, McBride TJ, Nichols GS, Pannoni KE, Sanculi D, Totten DJ (2014) Input clustering and the microscale structure of local circuits. Front Neural Circuits 8:112.

Denk W, Horstmann H (2004) Serial block-face scanning electron microscopy to reconstruct three-dimensional tissue nanostructure. PLoS Biol 2:e329.

Erişir A, Van Horn SC, Bickford ME, Sherman SM (1997) Immunocytochemistry and distribution of parabrachial terminals in the lateral geniculate nucleus of the cat: a comparison with corticogeniculate terminals. J Comp Neurol 377:535-549.

Feldman DE, Knudsen El (1997) An anatomical basis for visual calibration of the auditory space map in the barn owl's midbrain. J Neurosci 17:6820-6837.

Feldman DE, Brainard MS, Knudsen El (1996) Newly learned auditory responses mediated by NMDA receptors in the owl inferior colliculus. Science 271:525-528.

Fiala JC, Kirov SA, Feinberg MD, Petrak LJ, George P, Goddard CA, Harris KM (2003) Timing of neuronal and glial ultrastructure disruption during brain slice preparation and recovery in vitro. J Comp Neurol 465:90-103.

Grutzendler J, Kasthuri N, Gan WB (2002) Long-term dendritic spine stability in the adult cortex. Nature 420:812-816.

Harris KM, Stevens JK (1988) Dendritic spines of rat cerebellar Purkinje cells: serial electron microscopy with reference to their biophysical characteristics. J Neurosci 8:4455-4469.

Hell SW (2003) Toward fluorescence nanoscopy. Nat Biotechnol 21:1347-1355.

Hering H, Sheng M (2001) Dendritic spines: structure, dynamics and regulation. Nat Rev Neurosci 2:880-888.

Hyde PS, Knudsen El (2000) Topographic projection from the optic tectum to the auditory space map in the inferior colliculus of the barn owl. J Comp Neurol 421:146-160.

Jones EG, Powell TP (1969) Electron microscopy of synaptic glomeruli in the thalamic relay nuclei of the cat. Proc R Soc Lond B Biol Sci 172:153-171.

Kasthuri N, Hayworth KJ, Berger DR, Schalek RL, Conchello JA, Knowles-Barley S, Lee D, Vázquez-Reina A, Kaynig V, Jones TR, Roberts M, Morgan JL, Tapia JC, Seung HS, Roncal WG, Vogelstein JT, Burns R, Sussman DL, Priebe CE, Pfister H, Lichtman JW (2015) Saturated reconstruction of a volume of neocortex. Cell 162:648-661.

Knudsen El (2002) Instructed learning in the auditory localization pathway of the barn owl. Nature 417:322-328.

Knudsen El, Konishi M (1978) A neural map of auditory space in the owl. Science 200:795-797.

Konishi M (2003) Coding of auditory space. Annu Rev Neurosci 26:31-55.

Kwon T, Sakamoto M, Peterka DS, Yuste R (2017) Attenuation of synaptic potentials in dendritic spines. Cell Rep 20:1100-1110.

Lavenex P, Lavenex PB, Bennett JL, Amaral DG (2009) Postmortem changes in the neuroanatomical characteristics of the primate brain: hippocampal formation. J Comp Neurol 512:27-51.

Lee KJ, Kim H, Kim TS, Park SH, Rhyu IJ (2004) Morphological analysis of spine shapes of Purkinje cell dendrites in the rat cerebellum using high-voltage electron microscopy. Neurosci Lett 359:21-24.

Luksch H, Gauger B, Wagner H (2000) A candidate pathway for a visual instructional signal to the barn owl's auditory system. J Neurosci 20:RC70.

McBride TJ, DeBello WM (2015) Input clustering in the normal and learned circuits of adult barn owls. Neurobiol Learn Mem 121:3951

McBride TJ, Rodriguez-Contreras A, Trinh A, Bailey R, Debello WM (2008) Learning drives differential clustering of axodendritic contacts in the barn owl auditory system. J Neurosci 28:6960-6973.

Mishchenko Y, Hu T, Spacek J, Mendenhall J, Harris KM, Chklovski DB (2010) Ultrastructural analysis of hippocampal neuropil from the connectomics perspective. Neuron 67:1009-1020.

Morgan JL, Berger DR, Wetzel AW, Lichtman JW (2016) The fuzzy logic of network connectivity in mouse visual thalamus. Cell 165: 192-206.

Müller W, Connor JA (1991) Dendritic spines as individual neuronal compartments for synaptic Ca2 + responses. Nature 354:73-76.

Mysore SP, Knudsen El (2014) Descending control of neural bias and selectivity in a spatial attention network: rules and mechanisms. Neuron 84:214-226.

Niederleitner B, Luksch H (2012) Neuronal morphology in subdivisions of the inferior colliculus of chicken (Gallus gallus). J Chem Neuroanat 44:24-33.

Olsen JF, Knudsen El, Esterly SD (1989) Neural maps of interaural time and intensity differences in the optic tectum of the barn owl. J Neurosci 9:2591-2605.

Peña JL, Konishi M (2001) Auditory spatial receptive fields created by multiplication. Science 292:249-252.

Peña JL, Konishi M (2000) Cellular mechanisms for resolving phase ambiguity in the owl's inferior colliculus. Proc Natl Acad Sci USA 97:11787-11792.

Peña JL, Konishi M (2002) From postsynaptic potentials to spikes in the genesis of auditory spatial receptive fields. J Neurosci 22: 5652-5658.

Peña JL, Konishi M (2004) Robustness of multiplicative processes in auditory spatial tuning. J Neurosci 24:8907-8910.

Poirazi P, Brannon T, Mel BW (2003) Pyramidal neuron as two-layer neural network. Neuron 37:989-999.

Rall W (1974) Dendritic spines, synaptic potency and neuronal plasticity. Los Angeles: University of California.

Rapisardi SC, Miles TP (1984) Synaptology of retinal terminals in the dorsal lateral geniculate nucleus of the cat. J Comp Neurol 223: 515-534.

Rodriguez-Contreras A, Liu XB, DeBello WM (2005) Axodendritic contacts onto calcium/calmodulin-dependent protein kinase type II-expressing neurons in the barn owl auditory space map. J Neurosci 25:5611-5622.

Sabatini BL, Maravall M, Svoboda K (2001) $\mathrm{Ca}(2+)$ signaling in dendritic spines. Curr Opin Neurobiol 11:349-356.

Singheiser M, Gutfreund Y, Wagner H (2012) The representation of sound localization cues in the barn owl's inferior colliculus. Front Neural Circuits 6:45.

Sorra KE, Fiala JC, Harris KM (1998) Critical assessment of the involvement of perforations, spinules, and spine branching in hippocampal synapse formation. J Comp Neurol 398:225-240.

Spacek J, Lieberman AR (1974) Ultrastructure and three-dimensional organization of synaptic glomeruli in rat somatosensory thalamus. J Anat 117:487-516.

Stepanyants A, Chklovskii DB (2005) Neurogeometry and potential synaptic connectivity. Trends Neurosci 28:387-394.

Stepanyants A, Hof PR, Chklovskii DB (2002) Geometry and structural plasticity of synaptic connectivity. Neuron 34:275-288.

Takahashi TT, Carr CE, Brecha N, Konishi M (1987) Calcium binding protein-like immunoreactivity labels the terminal field of nucleus laminaris of the barn owl. J Neurosci 7:1843-1856.

Trachtenberg JT, Chen BE, Knott GW, Feng G, Sanes JR, Welker E, Svoboda K (2002) Long-term in vivo imaging of experiencedependent synaptic plasticity in adult cortex. Nature 420:788-794. 
Urban NN, Henze DA, Barrionuevo G (2001) Revisiting the role of the hippocampal mossy fiber synapse. Hippocampus 11:408-417.

Wagner H, Takahashi T, Konishi M (1987) Representation of interaural time difference in the central nucleus of the barn owl's inferior colliculus. J Neurosci 7:3105-3116.

Wagner H, Güntürkün O, Nieder B (2003) Anatomical markers for the subdivisions of the barn owl's inferior-collicular complex and adjacent peri- and subventricular structures. J Comp Neurol 465:145-159.

Wilke SA, Antonios JK, Bushong EA, Badkoobehi A, Malek E, Hwang M, Terada M, Ellisman MH, Ghosh A (2013) Decon- structing complexity: serial block-face electron microscopic analysis of the hippocampal mossy fiber synapse. J Neurosci 33:507-522.

Winkowski DE, Knudsen El (2006) Top-down gain control of the auditory space map by gaze control circuitry in the barn owl. Nature 439:336-339.

Yuste R (2013) Electrical compartmentalization in dendritic spines. Annu Rev Neurosci 36:429-449.

Zheng W, Knudsen El (1999) Functional selection of adaptive auditory space map by GABAA-mediated inhibition. Science 284:962-965. 